\title{
Principles of fuel ion ratio measurements in fusion plasmas by collective Thomson scattering
}

Stejner Pedersen, Morten; Nielsen, Stefan Kragh; Bindslev, Henrik; Korsholm, Søren Bang; Salewski, Mirko

Published in:

Plasma Physics and Controlled Fusion

Link to article, DOI:

$10.1088 / 0741-3335 / 53 / 6 / 065020$

Publication date:

2011

Document Version

Early version, also known as pre-print

Link back to DTU Orbit

Citation (APA):

Stejner Pedersen, M., Nielsen, S. K., Bindslev, H., Korsholm, S. B., \& Salewski, M. (2011). Principles of fuel ion ratio measurements in fusion plasmas by collective Thomson scattering. Plasma Physics and Controlled Fusion, 53(6), 065020. https://doi.org/10.1088/0741-3335/53/6/065020

\section{General rights}

Copyright and moral rights for the publications made accessible in the public portal are retained by the authors and/or other copyright owners and it is a condition of accessing publications that users recognise and abide by the legal requirements associated with these rights.

- Users may download and print one copy of any publication from the public portal for the purpose of private study or research.

- You may not further distribute the material or use it for any profit-making activity or commercial gain

- You may freely distribute the URL identifying the publication in the public portal 


\title{
Principles of fuel ion ratio measurements in fusion plasmas by collective Thomson scattering
}

\author{
M. Stejner, S. K. Nielsen, H. Bindslev, S. B. Korsholm, \\ M. Salewski \\ Association EURATOM-Ris $\varnothing$ DTU, DK-4000 Roskilde, Denmark \\ E-mail: mspe@risoe.dtu.dk
}

\begin{abstract}
For certain scattering geometries collective Thomson scattering (CTS) measurements are sensitive to the composition of magnetically confined fusion plasmas. CTS therefore holds the potential to become a new diagnostic for measurements of the fuel ion ratio - i.e., the tritium to deuterium density ratio. Measurements of the fuel ion ratio will be important for plasma control and machine protection in future experiments with burning fusion plasmas. Here we examine the theoretical basis for fuel ion ratio measurements by CTS. We show that the sensitivity to plasma composition is enhanced by the signatures of ion cyclotron motion and ion Bernstein waves which appear for scattering geometries with resolved wave vectors near perpendicular to the magnetic field. We investigate the origin and properties of these features in CTS spectra and give estimates of their relative importance for fuel ion ratio measurements.
\end{abstract}

25 March 2011

PACS numbers: 52.25.Os, 52.35.Hr, 52.40.Db, 52.70.Gw

Submitted to: Plasma Phys. Control. Fusion 


\section{Introduction}

In this paper we examine the theoretical basis for the use of collective Thomson scattering (CTS) to diagnose the density ratio between the fuel ion species in a magnetically confined fusion plasma. This so-called fuel ion ratio is of general scientific interest for fusion plasma experiments. The fuel ion ratio will further be a key parameter for machine protection and basic plasma control on next-step devices such as ITER where plasmas with significant fusion power are expected [1]. However, it is not clear if the fuel ion ratio can be determined in the plasma center $(\rho<0.3)$ with the diagnostic set currently included in the ITER baseline design. Therefore additional approaches are desired $[1,2]$. Microwave-based CTS diagnostics are well suited for reactor environments and provide access to the dynamics of confined ion populations by measuring the spectrum of probe radiation scattered by plasma fluctuations. The scattered radiation is picked up by a receiving antenna and the measurement is localized at the intersection of the probe and receiver beams. CTS diagnostics were originally developed to diagnose bulk plasma parameters such as ion temperatures [3-6], and they have since been applied to measurements of the velocity distributions of non-thermal energetic ion populations at JET, TEXTOR and ASDEX Upgrade [7-13]. For certain scattering geometries, CTS spectra also contain features which are highly sensitive to plasma composition [14]. It has been suggested $[15,16]$ that measurements of these features could form the basis for a new fuel ion ratio diagnostic.

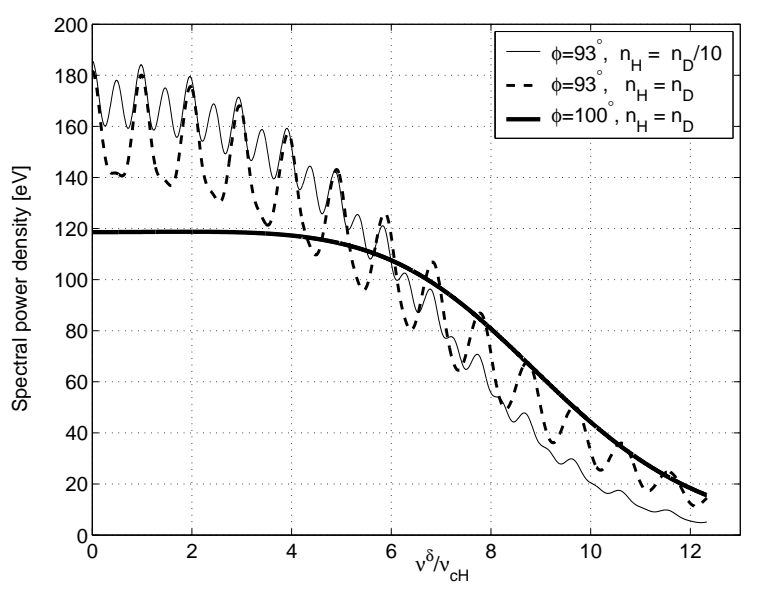

(a)

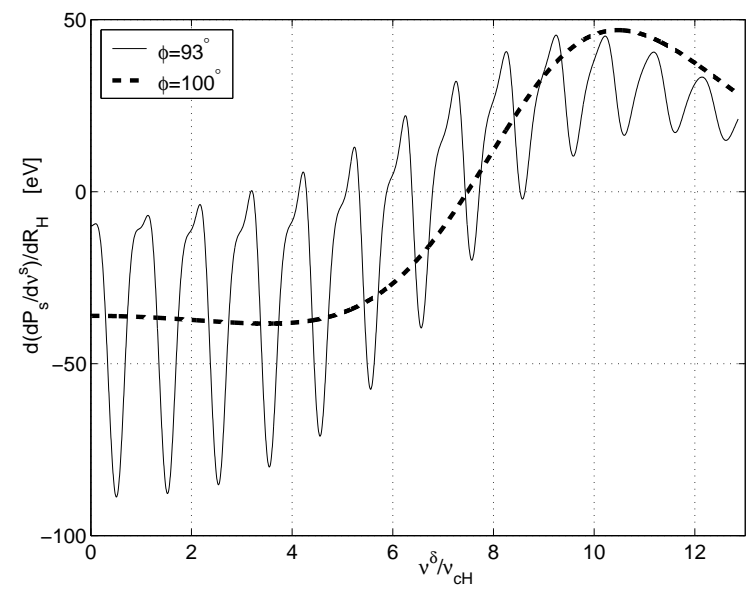

(b)

Figure 1. (a) Numerically calculated spectral power densities for different scattering geometries and plasma compositions. (b) Numerically calculated derivatives of the spectral power density with respect to the density ratio $R_{\mathrm{H}}=n_{\mathrm{H}} /\left(n_{\mathrm{H}}+n_{\mathrm{D}}\right)$. Plasma parameters and frequencies correspond to the plasma scenario defined in Appendix A. $\nu^{\delta}$ is the frequency shift of the scattered radiation, and the frequency scale is normalized by the hydrogen ion cyclotron frequency. For $\phi$ near $90^{\circ}$ the spectra show cyclotron structure which is highly sensitive to plasma composition. When $\phi$ is not close to $90^{\circ}$ (i.e., more than a few degrees away from $90^{\circ}$ ) the structure disappears and the spectrum is less sensitive to plasma composition. 
Figure 1 illustrates the basic idea. Figure 1a shows numerically calculated spectral power densities for frequencies and plasma parameters relevant to CTS experiments at TEXTOR. The model used to calculate these spectra will be discussed in detail in the following sections. The shape of the curves changes considerably if either the fuel ion ratio or the angle $\phi=\angle\left(\mathbf{k}^{\delta}, \mathbf{B}^{(0)}\right)$ resolved by the measurement is varied, keeping all other parameters constant. Here $\mathbf{k}^{\delta}$ is the plasma fluctuation wave vector resolved by the measurement, and $\mathbf{B}^{(0)}$ is the local magnetic field. When $\phi$ is close to $90^{\circ}$, the received radiation results from scattering of incident radiation off plasma fluctuations traveling almost perpendicularly to the magnetic field. For such scattering geometries peaks appear in the spectrum at intervals corresponding roughly to the cyclotron frequencies of the most common ions in the plasma - which were here taken to be hydrogen and deuterium. Thus, we note two sets of peaks in the spectra: one set which appears near hydrogen cyclotron harmonics and another set which appears near deuterium cyclotron harmonics for high deuterium density. When $\phi$ is more than a few degrees away from $90^{\circ}$ the peaks disappear, and the spectrum is less sensitive to plasma composition.

In CTS the incident probe radiation scatters off plasma fluctuations which are driven mainly by the thermal motion of ions in the plasma (externally driven fluctuations will not be considered here). In the following sections we shall see that the driving terms for these fluctuations contain an underlying cyclotron structure with contributions from each harmonic of the cyclotron motion of charged particles in the plasma. For fluctuations with wave vectors nearly perpendicular to the magnetic field, $\phi \sim 90^{\circ}$, the ion cyclotron motion dominates the driving terms which are strongly enhanced at frequencies coinciding with harmonics of the ion cyclotron frequency (or frequencies, in plasmas with multiple ion species). Plasma fluctuations result from the dielectric response of the plasma to the effects of the ion motion described by the driving terms. This response is particularly strong at frequencies and wave vectors corresponding to weakly damped plasma waves. In particular, weakly damped ion Bernstein waves strongly affect the spectrum of fluctuations with wave vectors nearly perpendicular to the magnetic field. The ion Bernstein waves have frequencies which lie between and often close to harmonics of the ion cyclotron frequencies, and the waves cause peaks in the fluctuation spectrum at these frequencies. The combined effect of the enhanced drive and plasma response at specific - but different - frequencies can be seen in the spectra in figure 1 for which $\phi$ is close to $90^{\circ}$. As noted above, these spectra contain peaks at frequencies close to the ion cyclotron harmonics. We refer to the peaks originating from this combined effect as ion cyclotron structure in the CTS spectrum.

Figure 1b shows the numerically calculated derivative of the spectral power density with respect to the density ratio $R_{\mathrm{H}}=n_{\mathrm{H}} /\left(n_{\mathrm{H}}+n_{\mathrm{D}}\right)$. The large-scale shapes of the spectra are determined mainly by the velocity distribution of the plasma ions. For increasing $R_{\mathrm{H}}$ the spectrum will broaden due to the higher thermal velocity of hydrogen ions relative to deuterium ions (assuming thermal equilibrium between the two ion species). The derivative with respect to $R_{\mathrm{H}}$ is therefore negative for small frequency shift and positive for large frequency shift as figure 1 illustrates. When $\phi$ is close to $90^{\circ}$ 
the ion cyclotron structure creates additional detailed structures in the derivative. In particular, the amplitude of the cyclotron structures - the peak amplitude accounting for the general slope of the spectrum - is highly sensitive to plasma composition. The widths and center frequencies of the peaks are also sensitive to $R_{\mathrm{H}}$, and in general the presence of the cyclotron structure strongly enhances the sensitivity of the spectrum to plasma composition. In addition, the higher level of detail in the derivative with respect to $R_{\mathrm{H}}$ has the effect that degeneracies of the functional dependence of the spectrum on plasma composition with the functional dependence on other plasma parameters can be resolved when $\phi$ is near $90^{\circ}$. It is this strong and detailed sensitivity which allows inference of the fuel ion ratio from a measured spectrum.

Indeed, previous feasibility studies [16] have found that such a diagnostic could fulfill the measurement requirements for ITER and that it could be combined with the CTS system foreseen to measure fast ion velocity distributions on ITER [17-19]. In preparation for proof-of-principle experiments the CTS receiver at TEXTOR was recently modified for measurements with frequency resolution better than $1 \mathrm{MHz}$ [20] as would be required to demonstrate the ability to resolve cyclotron structure in CTS spectra. The first measurements with the modified receiver demonstrating the ability to resolve ion cyclotron structure in the CTS spectrum were reported in [14]. The same series of experiments further demonstrated the sensitivity of the ion cyclotron structure to plasma composition with measurements taken in plasmas dominated by hydrogen, deuterium and ${ }^{3} \mathrm{He}$, respectively.

In this paper we examine the origin of cyclotron structure in CTS spectra theoretically. We illustrate our results with numerical examples, and to ensure the relevance of our numerical work to experiments possible on present machines, the examples are based on plasma parameters relevant to the CTS experiments at TEXTOR. For use in this paper we therefore define a standard plasma scenario relevant to CTS experiments at TEXTOR. The parameters for this scenario are given in Appendix A, and we use these parameters in all numerical calculations except where changes are explicitly noted in the text or in figure captions. In this scenario we consider plasmas consisting of fully ionized hydrogen and deuterium, and we investigate the sensitivity of the spectrum to the hydrogen to deuterium density ratio $R_{\mathrm{H}}=n_{\mathrm{H}} /\left(n_{\mathrm{D}}+n_{\mathrm{H}}\right)$. The sensitivity of the spectrum to $R_{\mathrm{H}}$ is entirely analogous to the sensitivity to the fuel ion ratio in a DT-plasma and we can use $R_{\mathrm{H}}$ as a proxy for the fuel ion ratio with no loss of generality.

Section 2 gives an outline of the model used in the numerical calculations. In Sections. 3 and 4 we show that the ion cyclotron structure results partly from the direct influence of ion cyclotron motion on the driving terms for the plasma fluctuations and partly from the influence of weakly damped ion Bernstein waves on the thermal fluctuation spectrum. In section 5 we examine the relative importance of the two effects in the total spectrum for the purpose of fuel ion ratio diagnostics. Here we find that both effects are generally present in CTS spectra with $\phi \sim 90^{\circ}$ and that for certain parameter ranges both effects are required for accurate determination of the fuel ion 
ratio.

\section{Kinetic model of collective Thomson Scattering}

In CTS measurements an incident macroscopic probing wave with wave vector and angular frequency $\left(\mathbf{k}^{\mathrm{i}}, \omega^{\mathrm{i}}\right)$ interacts with microscopic thermal fluctuations in the plasma with wave vector and frequency $\left(\mathbf{k}^{\delta}, \omega^{\delta}\right)$. The interaction sets up a current which in turn drives a third wave, the scattered wave with wave vector and frequency $\left(\mathbf{k}^{\mathrm{s}}=\mathbf{k}^{\mathrm{i}}+\mathbf{k}^{\delta}, \omega^{\mathrm{s}}=\omega^{\mathrm{i}}+\omega^{\delta}\right)$. When detected, the scattered wave allows inference of plasma properties which affect the thermal fluctuations that caused the scattering. In this paper the scattering process will be described using the model developed in [21-24]. The scattering process is treated in a fully electromagnetic approach assuming that the plasma response can be considered cold at the frequencies and wave vectors of the incident and scattered waves and that effects of collisions are negligible. The treatment of the fluctuations assumes a homogeneous plasma, but no further assumptions are made about the nature of the fluctuations which are described in a fully kinetic approach. The model is therefore not limited to cold collective fluctuations, and it will include the effects of warm plasma fluctuations such as ion Bernstein waves which enter the spectrum when the resolved fluctuation wave vector is near perpendicular to the main magnetic field in the plasma. In [23] it is demonstrated that for $\phi \sim 90^{\circ}$ scattering from fluctuations in quantities other than the electron density, as well as the relative phase of these components, may play a significant role. For such geometries a fully electromagnetic approach is therefore generally required to describe the scattering process.

Using the compact formulation given in [23] the received spectral power density is given by $\ddagger$

$$
\begin{aligned}
& \frac{\partial P^{\mathrm{s}}}{\partial \omega_{\mathrm{s}}}=P^{\mathrm{i}} O_{\mathrm{b}}\left(\lambda_{0}^{\mathrm{i}}\right)^{2} r_{\mathrm{e}}^{2} \frac{1}{2 \pi} \sum_{\mathrm{a}} \Sigma^{(\mathrm{a})}, \text { with a }=\text { electrons, ion species } \\
& \Sigma^{(\mathrm{a})}=\sum_{\alpha \beta} \Sigma_{\alpha \beta}^{(\mathrm{a})}, \text { with } \alpha, \beta=E, B, j, n .
\end{aligned}
$$

Here $\Sigma_{\alpha \beta}^{(a)}$ is the scattering function for plasma fluctuations in the field and fluid variables $(\alpha, \beta)$ driven by the thermal motion of particle species $a$. The indices $\alpha$ and $\beta$ represent the fluctuating quantities which are relevant to the scattering process, namely, the electron density, $n$, and the electron current, $j$, as well as the electric and magnetic fields. For simplicity $n$ and $j$ do not carry a superscript indicating particle species when used in this capacity. $P^{\mathrm{i}}$ is the power in the incident probing beam, $O_{\mathrm{b}}$ is the beam overlap, $\lambda_{0}^{\mathrm{i}}=\omega^{\mathrm{i}} / c$ is the vacuum wavelength of the probing radiation, and $r_{\mathrm{e}}=q_{\mathrm{e}}^{2} / 4 \pi \epsilon_{0} m_{\mathrm{e}} c^{2}$ is the classical electron radius. The beam overlap is defined as the volume integral over the product of the normalized probe and receiver beam intensities. The beam intensities

$\ddagger$ Note that these expressions are different from those in [23] in two respects: The front factor in equation (1) is not proportional to the unperturbed density (which is contained in $\Sigma^{(a)}$ ) and the summation over particle species is made explicit. 
will usually be Gaussian, but some insight can be gained by noting that for uniform beam intensities and perfect intersection the beam overlap is given by the ratio of the scattering volume to the product of the beam cross-sectional areas, $O_{\mathrm{b}} \simeq V / A^{\mathrm{i}} A^{\mathrm{s}}$. Due to refraction $O_{\mathrm{b}}$ will have some minor frequency dependence which is ignored here. The spectral variation of the received scattered power is contained in the scattering functions which are given by

$$
\Sigma_{\alpha \beta}^{(a)}=\frac{\left(\omega^{\mathrm{i}} \omega^{\mathrm{s}}\right)^{2}}{\omega_{\mathrm{pe}}^{4}} \frac{1}{\mathcal{L}^{\mathrm{i}} \mathcal{L}^{\mathrm{s}}} \hat{\mathbf{G}}_{i}^{(\alpha)}\left\langle\tilde{\alpha}_{i}^{(\mathrm{a})} \tilde{\beta}_{j}^{(\mathrm{a})}\right\rangle \hat{\mathbf{G}}_{j}^{(\beta)^{*}}
$$

Here $\omega_{\text {pe }}$ is the electron plasma frequency, and $\mathcal{L}^{\mathrm{i}}$ and $\mathcal{L}^{\mathrm{s}}$ are the normalized fluxes of the incident and scattered radiation, respectively. Summation of repeated lower indices is implied. An overhead tilde indicates thermal fluctuation levels, so for instance $\tilde{\mathbf{B}}=\mathbf{B}^{\mathrm{M}}-\mathbf{B}$ where $\mathbf{B}^{\mathrm{M}}$ is the magnetic field of the microscopic plasma state and $\mathbf{B}=\left\langle\mathbf{B}^{\mathrm{M}}\right\rangle$ is the macroscopic magnetic field given by the ensemble average of microscopic states. The coordinate system is defined such that $\mathbf{B}=B \hat{\mathbf{z}}$ and $\mathbf{k}^{\delta}=k_{\|}^{\delta} \hat{\mathbf{z}}+k_{\perp}^{\delta} \hat{\mathbf{x}}$

The dielectric coupling operators, $\hat{\mathbf{G}}_{i}^{(\alpha)}$, describe interaction of the incident wave with fluctuations in the set of field and fluid variables $\left\{E_{i}, B_{i}, j_{i}, n\right\}$ and the coupling to the scattered wave. Explicit expressions for the normalized fluxes and dielectric coupling operators are derived in $[21,22]$ and given in a compact notation in [23], and they will not be reproduced here. While these factors are of great importance for the scattering theory, they impart little spectral variation, and therefore little sensitivity to plasma composition, to the received scattered power. Rather, our attention shall be focused on the Fourier transform of the correlation of fluctuations in the field and fluid quantities, $\left\langle\tilde{\alpha}_{i}^{(\mathrm{a})} \tilde{\beta}_{j}^{(\mathrm{a})}\right\rangle$, which contains most of the sensitivity to plasma composition. We shall give expressions for these terms below, but for details of their derivation we refer to [23,24].

In the dressed particle approach $\left\langle\tilde{\alpha}_{i}^{(\mathrm{a})} \tilde{\beta}_{j}^{(\mathrm{a})}\right\rangle$ can be calculated as the product between the correlation tensor for unscreened current fluctuations in particle species $a,\left\langle\tilde{\mathbf{j}}^{(\mathrm{a} 0)} \tilde{\mathbf{j}}^{(\mathrm{a} 0)}\right\rangle$, and the corresponding fluctuation operators,

$$
\left\langle\tilde{\alpha}_{i}^{(\mathrm{a})} \tilde{\beta}_{j}^{(\mathrm{a})}\right\rangle=\hat{\mathbf{S}}_{i k}^{(\alpha \mathrm{a})}\left\langle\tilde{j}_{k}^{(\mathrm{a} 0)} \tilde{j}_{l}^{(\mathrm{a} 0)}\right\rangle \hat{\mathbf{S}}_{j l}^{(\beta \mathrm{a}) *} .
$$

Here the fluctuation operators, $\hat{\mathbf{S}}_{i k}^{(\alpha a)}$, quantify the plasma response, $\tilde{\alpha}_{i}^{(\mathrm{a})}$, in the quantity $\alpha$ to unscreened current fluctuations in particle species $a$,

$$
\tilde{\alpha}_{i}=\sum_{\mathrm{a}} \tilde{\alpha}_{i}^{(\mathrm{a})} \text { with } \tilde{\alpha}_{i}^{(\mathrm{a})}=\hat{\mathbf{S}}_{i k}^{(\alpha \mathrm{a})} \tilde{j}_{k}^{(a 0)}
$$

The fluctuation operators may be derived from the plasma wave equation, and explicit expressions for each operator will be given in section. 4. The unscreened current correlation tensor is given by

$$
\left\langle\tilde{j}_{k}^{(\mathrm{a} 0)} \tilde{j}_{k^{\prime}}^{(\mathrm{a} 0)}\right\rangle=q_{\mathrm{a}}^{2} \int v_{k} v_{k^{\prime}}\left\langle\tilde{f}^{(\mathrm{a} 0)}(\mathbf{p}) \tilde{f}^{(\mathrm{a} 0)}\left(\mathbf{p}^{\prime}\right)\right\rangle \mathrm{d} \mathbf{p} \mathrm{d} \mathbf{p}^{\prime} .
$$

where $\left\langle\tilde{f}^{(\mathrm{a} 0)}(\mathbf{p}) \tilde{f}^{(\mathrm{a} 0)}\left(\mathbf{p}^{\prime}\right)\right\rangle$ is the spatial and temporal Fourier transform of the two time correlation $\left\langle\tilde{f}^{(\mathrm{a} 0)}(x, t) \tilde{f}^{(\mathrm{a} 0)}\left(x^{\prime}, t^{\prime}\right)\right\rangle$ and $q_{\mathrm{a}}$ is the charge of species $a$. Here 
$\tilde{f}^{(\mathrm{a})}=\tilde{f}^{(\mathrm{a} 0)}+\tilde{f}^{(\mathrm{a} 1)}$, where $\tilde{f}^{(\mathrm{a} 0)}$ represents the evolution of the microscopic distribution function $\tilde{f}^{(\mathrm{a})}$ in the absence of particle interactions and $\tilde{f}^{(\mathrm{a} 1)}$ represents the dielectric response of the plasma to the microscopic fields $(\tilde{\mathbf{E}}, \tilde{\mathbf{B}})$ set up by the unscreened particle motion. Similarly, $\left\langle\tilde{\alpha}_{i}^{(\mathrm{a})} \tilde{\beta}_{j}^{(\mathrm{a})}\right\rangle$ represents the plasma dielectric response to the free streaming particle motion described by the unscreened current correlation tensor $\left\langle\tilde{\mathbf{j}}^{(\mathrm{a} 0)} \tilde{\mathbf{j}}^{(\mathrm{a} 0)}\right\rangle$. To give an example, $\left\langle\tilde{n}^{(\mathrm{H})} \tilde{n}^{(\mathrm{H})}\right\rangle$ describes fluctuations in the electron density due to electron screening of hydrogen ions moving through the plasma.

The unscreened current correlation tensor, $\left\langle\tilde{\mathbf{j}}^{(\mathrm{a} 0)} \tilde{\mathbf{j}}^{(\mathrm{a} 0)}\right\rangle$, can be expressed in terms of the unperturbed macroscopic distribution function $[23,24]$

$$
\left\langle\tilde{\mathbf{j}}^{(\mathrm{a} 0)} \tilde{\mathbf{j}}^{(\mathrm{a} 0)}\right\rangle=(2 \pi)^{2} \frac{m_{\mathrm{a}} q_{\mathrm{a}}^{2}}{\left|k_{\|}\right|} \int \mathrm{d} p_{\perp} p_{\perp} \sum_{l=-\infty}^{\infty} \mathbf{c}_{l} \mathbf{c}_{l}^{*} f^{(a 0)}\left(p_{\perp}, p_{\|}\right)
$$

where

$$
\mathbf{c}_{l}=\left\{\begin{array}{c}
\frac{l \omega_{c a}}{k_{\perp}} J_{l}\left(k_{\perp} \rho_{\mathrm{a}}\right) \\
-i v_{\perp} J_{l}^{\prime}\left(k_{\perp} \rho_{\mathrm{a}}\right) \\
v_{\|} J_{l}\left(k_{\perp} \rho_{\mathrm{a}}\right)
\end{array}\right\}, \quad v_{\|}=\frac{\omega-l \omega_{\mathrm{ca}}}{k_{\|}}=\frac{p_{\|}}{m_{\mathrm{a}}}
$$

and $J_{l}$ are Bessel functions of the first kind of order $l$, primes indicate derivatives, $\omega_{\mathrm{ca}}=q_{\mathrm{a}} B^{(0)} / m_{\mathrm{a}}$ is the cyclotron frequency and $\rho_{\mathrm{a}}=v_{\perp} / \omega_{\mathrm{ca}}$ is the Larmor radius.

Below we shall see that for scattering geometries where the resolved fluctuation wave vector is nearly perpendicular to the unperturbed magnetic field, $\phi=\angle\left(\mathbf{B}^{(0)}, \mathbf{k}^{\delta}\right) \sim 90^{\circ}$, both $\left\langle\tilde{\mathbf{j}}^{(\mathrm{a} 0)} \tilde{\mathbf{j}}^{(\mathrm{a} 0)}\right\rangle$ and the fluctuation operators contain cyclotron structures which enhance the sensitivity of the total spectrum to plasma composition - as well as to certain other parameters such as the ion temperature. In the following sections we examine the origin and properties of the cyclotron structure in each term and then evaluate their relative importance for the total spectrum.

\section{Signatures of ion cyclotron motion in the unscreened current correlation tensor}

Assuming the unperturbed momentum distribution for particle species $a$ to be an isotropic Maxwellian - the only case to be considered here - with temperature $T_{\mathrm{a}}$ and particle density $n^{(\mathrm{a} 0)}$

$$
f^{(\mathrm{a} 0)}\left(p_{\perp}, p_{\|}\right)=\frac{n^{(\mathrm{a} 0)}}{\left(2 \pi m_{\mathrm{a}} T_{\mathrm{a}}\right)^{3 / 2}} \exp \left\{-\frac{p_{\perp}^{2}+p_{\|}^{2}}{2 m_{\mathrm{a}} T_{\mathrm{a}}}\right\}
$$

the unscreened current correlation tensor, equation (7), becomes

$$
\begin{aligned}
& \left\langle\tilde{\mathbf{j}}^{(a 0)} \tilde{\mathbf{j}}^{(a 0)}\right\rangle(\mathbf{k}, \omega)=\frac{(2 \pi)^{2} q_{a}^{2} n^{(a 0)} m_{\mathrm{a}}}{\left(2 \pi m_{\mathrm{a}} T_{\mathrm{a}}\right)^{3 / 2}\left|k_{\|}\right|} \sum_{l=-\infty}^{\infty} \exp \left\{-\zeta_{l}^{2}\right\} \mathbf{M}^{(l)} \\
& \mathbf{M}^{(l)}=\int d p_{\perp} p_{\perp} \mathbf{c}_{l} \mathbf{c}_{l}^{*} \exp \left\{-\frac{p_{\perp}^{2}}{2 m_{\mathrm{a}} T_{\mathrm{a}}}\right\}
\end{aligned}
$$




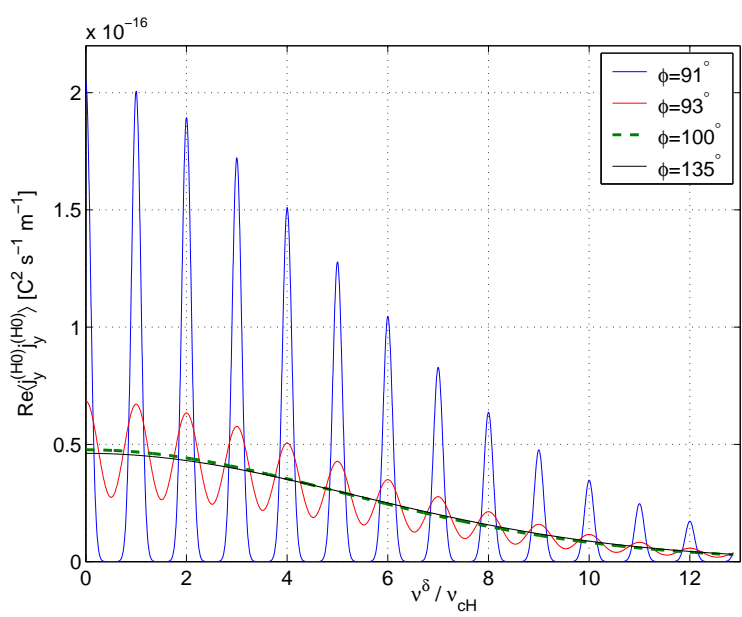

(a)

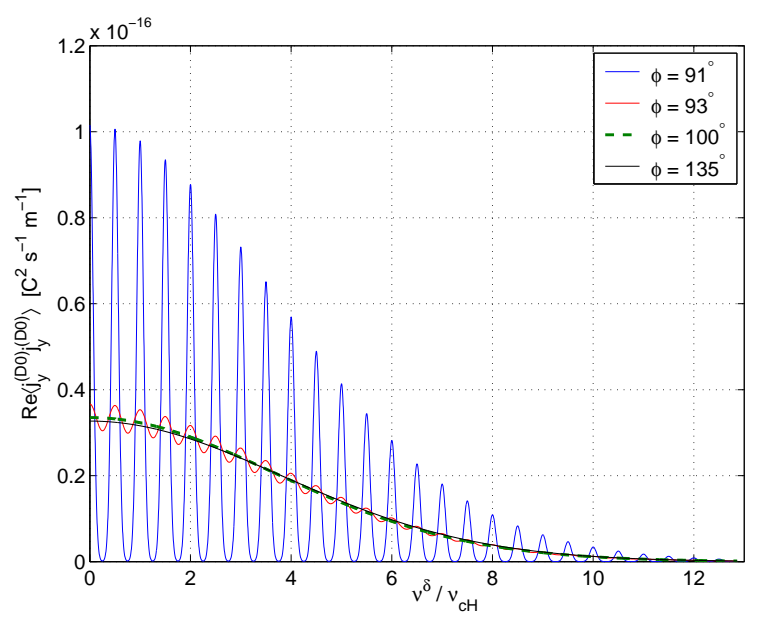

(b)

Figure 2. The $\left\langle\tilde{j}_{y}^{(\mathrm{H} 0)} \tilde{j}_{y}^{(\mathrm{H} 0)}\right\rangle(a)$ and $\left\langle\tilde{j}_{y}^{(\mathrm{D} 0)} \tilde{j}_{y}^{(\mathrm{D} 0)}\right\rangle(b)$ elements of the unscreened current correlation tensor for different values of $\phi=\angle\left(\mathbf{B}, \mathbf{k}^{\delta}\right)$. The frequency scale is normalized by the hydrogen cyclotron frequency. $\left\langle\tilde{\mathbf{j}}^{(\mathrm{i} 0)} \tilde{\mathbf{j}}^{(\mathrm{i} 0)}\right\rangle$ is even in $\nu^{\delta}$ for isotropic momentum distributions, so the elements are shown only for positive $\nu^{\delta}$. For $\phi$ close to $90^{\circ}$ peaks develop at each harmonic of the ion cyclotron frequency and for $\phi \rightarrow 90^{\circ}$ these elements go to zero except at the cyclotron harmonics.

with

$$
\zeta_{l}=\sqrt{\frac{m_{\mathrm{a}}}{2 T_{\mathrm{a}}}}\left(\frac{\omega-l \omega_{\mathrm{ca}}}{k_{\|}}\right)=\frac{v_{\|}}{v_{\mathrm{ta}}}, \quad v_{\mathrm{ta}}=\sqrt{2 T_{\mathrm{a}} / m_{\mathrm{a}}} .
$$

The momentum integrals in $\mathbf{M}^{(l)}$ can be solved analytically, and the sums over $l$ can be evaluated numerically by Clenshaw's method. Below, we shall examine numerical results for some elements in $\left\langle\tilde{\mathbf{j}}^{(\mathrm{i} 0)} \tilde{\mathbf{j}}^{(\mathrm{i} 0)}\right\rangle$, but the explicit forms will not be listed except for the $\left\langle\tilde{j}_{x}^{(\mathrm{i} 0)} \tilde{j}_{x}^{(\mathrm{i} 0)}\right\rangle$ element. We use the superscript $i$ to indicate any ion species, and below we shall use $I$ to indicate a sum over all ion species. We further note that while the diagonal elements of $\left\langle\tilde{\mathbf{j}}^{(\mathrm{a} 0)} \tilde{\mathbf{j}}^{(\mathrm{a} 0)}\right\rangle$ are real, the off-diagonal elements are in general complex quantities. However, $\Sigma_{\alpha \beta}^{(\mathrm{a})}$ is Hermitian when considered as a matrix with indices $(\alpha, \beta)$, so the sums $\Sigma_{\alpha \beta}^{(\mathrm{a})}+\Sigma_{\beta \alpha}^{(\mathrm{a})}$ are real. For simplicity we shall here restrict ourselves to examining the diagonal elements which are usually the dominant terms. This approach is useful to gain insight in the behavior of the unscreened current correlation tensor, but in calculations of the total scattering spectra we use the full expressions with all terms included.

Figure 2 shows examples of numerically calculated elements of $\left\langle\tilde{\mathbf{j}}^{(\mathrm{H} 0)} \tilde{\mathbf{j}}^{(\mathrm{H} 0)}\right\rangle$ and $\left\langle\tilde{\mathbf{j}}^{(\mathrm{D} 0)} \tilde{\mathbf{j}}^{(\mathrm{D} 0)}\right\rangle$ for different values of $\phi$ and for parameters corresponding to the standard scenario defined in Appendix A. When the resolved fluctuation wave vector component is nearly perpendicular to the magnetic field (i.e., in the limit where $\phi \rightarrow 90^{\circ}, k_{\|} \rightarrow 0$ and $\left.k_{\perp} \rightarrow k\right)$, the argument of the exponential function in equation (10) goes to negative infinity except at (or near) frequencies coinciding with a cyclotron harmonic where $\omega=l \omega_{\text {ca }}$ so $\zeta_{l}=0$. Here we are interested in the ion dynamics and plasma composition, 
so we focus our attention on the ion cyclotron range of frequencies and will not consider what happens near electron cyclotron harmonics. At each ion cyclotron harmonic, $\omega_{\text {ci }}$, there will be one term for which the exponential function in the sum over $l$ in equation (10) is unity. The elements of $\left\langle\tilde{\mathbf{j}}^{(\mathrm{i})} \tilde{\mathbf{j}}^{(\mathrm{i} 0)}\right\rangle$ in which the z-element of $\mathbf{c}_{l}$ is not involved then diverge due to the $\left|k_{\|}\right|^{-1}$ dependence in the front factor of equation (10). At other frequencies all terms in the sum over $l$ will be suppressed by the exponential function. Thus

$$
\lim _{\phi \rightarrow 90^{\circ}}\left\langle\tilde{j}_{k}^{(\mathrm{i} 0)} \tilde{j}_{k^{\prime}}^{(\mathrm{i} 0)}\right\rangle=\left\{\begin{array}{ll}
\infty & \text { for } \omega=n \omega_{c i} \\
0 & \text { for } \omega \neq n \omega_{c i}
\end{array} \quad n \in Z, \quad k, k^{\prime}=x, y\right.
$$

This behavior is seen in figure 2 as the gradual emergence at each ion cyclotron harmonic of peaks with increasing amplitude and decreasing width for $\phi \rightarrow 90^{\circ}$ until finally these elements of $\left\langle\tilde{\mathbf{j}}^{(\mathrm{i} 0)} \tilde{\mathbf{j}}^{(\mathrm{i} 0)}\right\rangle$ consists of a series of delta function-like spikes (though note that such a spectrum is not obtained experimentally because the influence of collisions will ensure that the peaks reduce to a Lorentzian form [25]). These peaks are the signatures of ion cyclotron motion and will be referred to as ICM signatures. As seen in figure 2 the frequency separation between ICM signatures in $\left\langle\tilde{\mathbf{j}}^{(\mathrm{D} 0)} \tilde{\mathbf{j}}^{(\mathrm{D} 0)}\right\rangle$ is half that of $\left\langle\tilde{\mathbf{j}}^{(\mathrm{H} 0)} \tilde{\mathbf{j}}^{(\mathrm{H} 0)}\right\rangle$ corresponding to the ratio between the ion cyclotron frequencies for the two ion species. In this limit the shape of $\left\langle\tilde{\mathbf{j}}^{(\mathrm{i} 0)} \tilde{\mathbf{j}}^{(\mathrm{i} 0)}\right\rangle$ therefore depends strongly on the cyclotron frequency for the ion species in question. Figure 2 shows the $y y$-elements of $\left\langle\tilde{\mathbf{j}}^{(\mathrm{i} 0)} \tilde{\mathbf{j}}^{(\mathrm{i} 0)}\right\rangle$. Qualitatively similar behaviors are found for the $x x$-element, which will be examined in detail below, and for the off-diagonal elements, which will not be examined in detail.

In the opposite case, at values of $\phi$ far from $90^{\circ}$, each term in the sum over cyclotron harmonics contributes to the sum over a wide range of frequencies roughly centered around the cyclotron harmonic. The structure created in $\left\langle\tilde{\mathbf{j}}^{(\mathrm{i} 0)} \tilde{\mathbf{j}}^{(\mathrm{i} 0)}\right\rangle$ by the individual terms is then smeared out by contributions from other terms leaving the total spectrum with no noticeable signatures of ion cyclotron motion. The shape of $\left\langle\tilde{\mathbf{j}}^{(\mathrm{i} 0)} \tilde{\mathbf{j}}^{(\mathrm{i} 0)}\right\rangle$ then depends mainly on the thermal velocity of the ion species.

The behavior of elements in $\left\langle\tilde{\mathbf{j}}^{(\mathrm{i} 0)} \tilde{\mathbf{j}}^{(\mathrm{i} 0)}\right\rangle$ which involve the z-element of $\mathbf{c}_{l}$ is somewhat different because the z-element of $\mathbf{c}_{l}$ is proportional to $v_{\|}$and $v_{\|}=0$ at the ion cyclotron harmonics (see equation (8)). In the limit $\phi \rightarrow 90^{\circ}$ these elements therefore go to zero even at the cyclotron harmonics. This behavior is illustrated in figure 3 which shows $\left\langle\tilde{j}_{z}^{(\mathrm{H} 0)} \tilde{j}_{z}^{(\mathrm{H} 0)}\right\rangle$ for $\phi$ close to $90^{\circ}$ and the total $\left\langle\tilde{j}_{z}^{(\mathrm{I} 0)} \tilde{j}_{z}^{(\mathrm{I} 0)}\right\rangle$ element in a narrow frequency range around an ion cyclotron harmonic. When $\phi$ is very close to $90^{\circ}$ we find a double peaked structure in the vicinity of the ion cyclotron harmonic. This double peaked structure can be understood as a single peak centered on each cyclotron frequency, but with a hollow center due to the z-element of $\mathbf{c}_{l}$. At intermediate values of $\phi$ the structures broaden and eventually merge to form a single peak between the cyclotron harmonics.

For diagnostic purposes it is noteworthy that the ion cyclotron features in $\left\langle\tilde{\mathbf{j}}^{(\mathrm{i} 0)} \tilde{\mathbf{j}}^{(\mathrm{i} 0)}\right\rangle$ are highly sensitive to the strength and direction of the magnetic field as well as the ion thermal velocity. They also provide increased sensitivity to the plasma composition for scattering geometries where the cyclotron features are noticeable. Figure 4 illustrates 


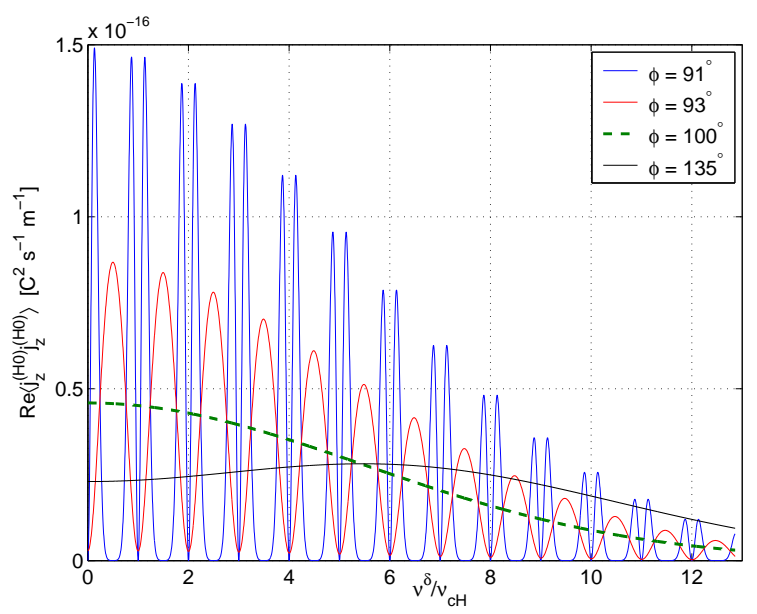

(a)

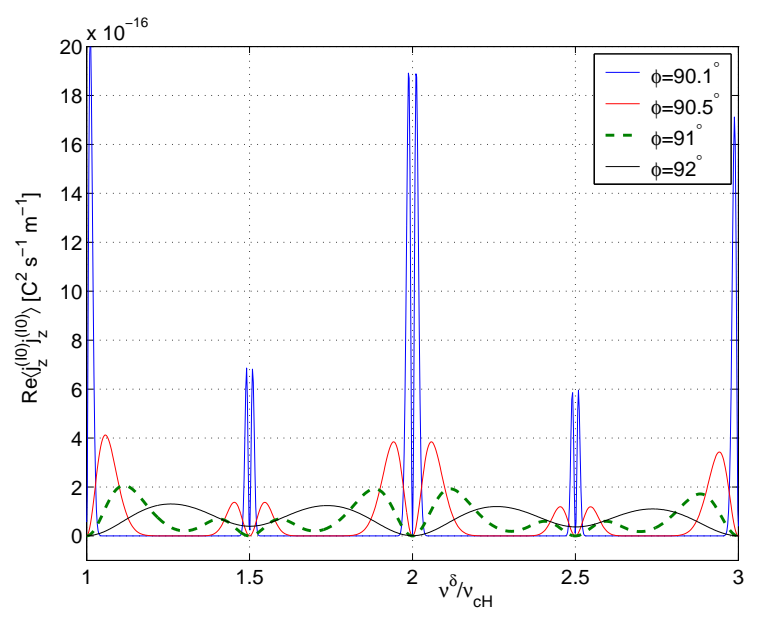

(b)

Figure 3. The $\left\langle\tilde{j}_{z}^{(\mathrm{H} 0)} \tilde{j}_{z}^{(\mathrm{H} 0)}\right\rangle$ element $(a)$ and the total $\left\langle\tilde{j}_{z}^{(\mathrm{I} 0)} \tilde{j}_{z}^{(\mathrm{I} 0)}\right\rangle=\left\langle\tilde{j}_{z}^{(\mathrm{H} 0)} \tilde{j}_{z}^{(\mathrm{H} 0)}\right\rangle+$ $\left\langle\tilde{j}_{z}^{(\mathrm{D} 0)} \tilde{j}_{z}^{(\mathrm{D} 0)}\right\rangle$ element $(b)$ of the unscreened current correlation tensor for different values of $\phi=\angle\left(\mathbf{B}, \mathbf{k}^{\delta}\right)$. The frequency scale is normalized by the hydrogen cyclotron frequency, and $(b)$ is focused around a single hydrogen cyclotron harmonic. A double peaked structures is found for $\phi$ very close to $90^{\circ}$. The structure broadens for angles further from perpendicular and at $\phi=92^{\circ}$ the peaks have merged to form a single peak between cyclotron harmonics.

this sensitivity with elements of $\left\langle\tilde{\mathbf{j}}^{(\mathrm{I} 0)} \tilde{\mathbf{j}}^{(\mathrm{I} 0)}\right\rangle$ calculated at different compositions and geometries and with contributions to $\left\langle\tilde{\mathbf{j}}^{(\mathrm{I} 0)} \tilde{\mathbf{j}}^{(\mathrm{I} 0)}\right\rangle$ from different ion species. We shall discuss the origins and properties of this sensitivity as well as some limitations to its usefulness for diagnostics purposes.

Contributions to $\left\langle\tilde{\mathbf{j}}^{(\mathrm{I0})} \tilde{\mathbf{j}}^{(\mathrm{I} 0)}\right\rangle$ are linearly proportional to the density of each ion species through the front factor in equation (10), but their shapes do not depend directly on the ion densities. For given wave vectors and magnetic field strength, the shape of $\left\langle\tilde{\mathbf{j}}^{(\mathrm{i} 0)} \tilde{\mathbf{j}}^{(\mathrm{i} 0)}\right\rangle$ depends on the ion cyclotron frequency (i.e., the charge to mass ratio) and the ion thermal velocity (i.e., the mass and temperature of each ion species). If two ion species have the same density, thermal velocity and cyclotron frequency (i.e., $T_{1} / m_{1}=T_{2} / m_{2}$ and $\left.q_{1} / m_{1}=q_{2} / m_{2}\right)$ their contributions to $\left\langle\tilde{\mathbf{j}}^{(\mathrm{I} 0)} \tilde{\mathbf{j}}^{(\mathrm{I} 0)}\right\rangle$ will differ only by a constant factor $q_{1}^{2} / q_{2}^{2}$. In this case, variations in the density ratio result only in scaling of $\left\langle\tilde{\mathbf{j}}^{(\mathrm{I} 0)} \tilde{\mathbf{j}}^{(\mathrm{I} 0)}\right\rangle$. This is not useful for diagnostic purposes since such a scaling will be degenerate with a number of other experimental parameters (most notably the power in the probing beam and the quality of the beam overlap). However, in the more common situation that the ion thermal velocities are unequal this degeneracy is broken and the shape of $\left\langle\tilde{\mathbf{j}}^{(\mathrm{I0})} \tilde{\mathbf{j}}^{(\mathrm{I} 0)}\right\rangle$ will be sensitive to plasma composition. If the ions are in thermal equilibrium the shapes of contributions from ions with different masses will differ because the thermal velocities of heavier ions are lower resulting in more narrow contributions to $\left\langle\tilde{\mathbf{j}}^{(\mathrm{I0})} \tilde{\mathbf{j}}^{(\mathrm{I0})}\right\rangle$ and vice versa for lighter ions. This type of sensitivity to plasma composition does not depend on the presence of ICM signatures, and it provides the CTS spectrum with a weak sensitivity to the fuel ion ratio even for $\phi$ far from $90^{\circ}$. 


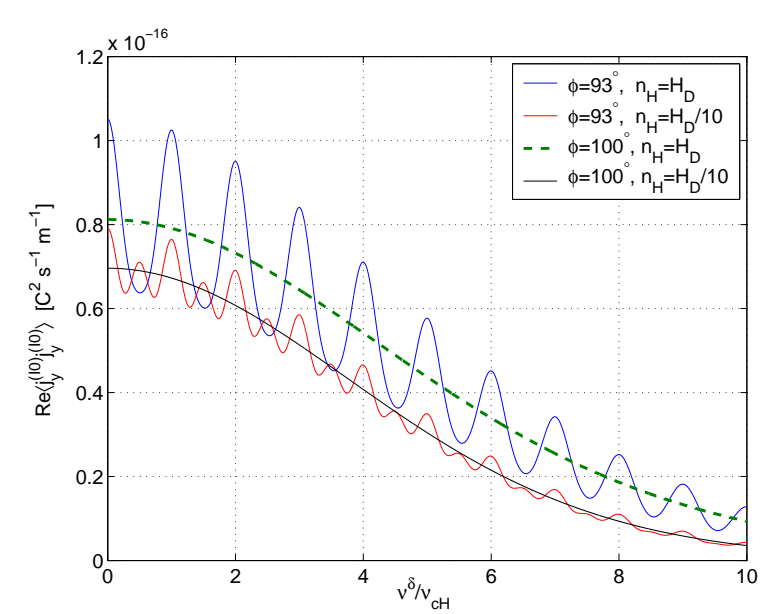

(a)

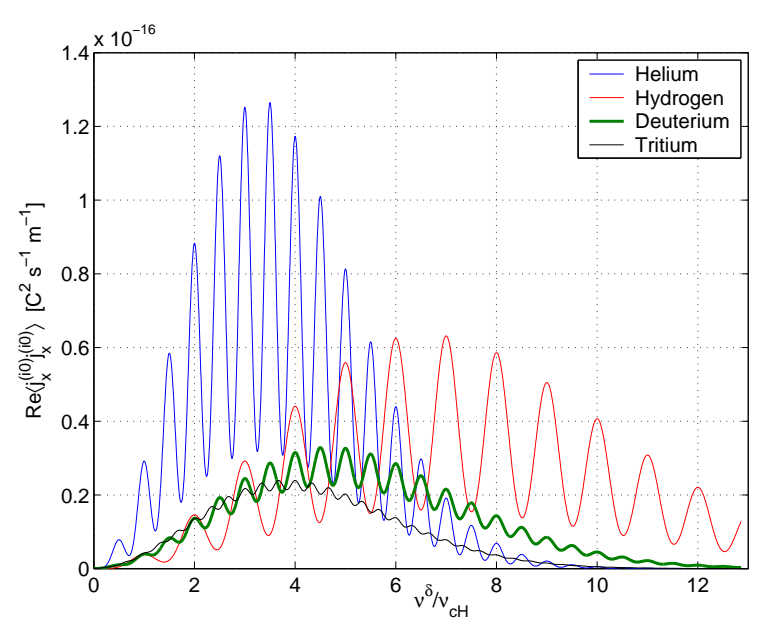

(b)

Figure 4. (a) Total $\left\langle\tilde{j}_{y}^{(\mathrm{I} 0)} \tilde{j}_{y}^{(\mathrm{I} 0)}\right\rangle$ elements for the standard plasma scenario, but with different density ratios for hydrogen and deuterium and at different values of $\phi$. (b) The $\left\langle\tilde{j}_{x}^{(\mathrm{i} 0)} \tilde{j}_{x}^{(\mathrm{i} 0)}\right\rangle$ elements for helium, hydrogen, deuterium and tritium. Plasma parameters as in the standard scenario but with $n_{\mathrm{e}}=5 \times 10^{-19} \mathrm{~m}^{-3}, n_{\mathrm{He}}=n_{\mathrm{H}}=n_{\mathrm{D}}=n_{\mathrm{T}}=$ $1 \times 10^{-19} \mathrm{~m}^{-3}$ and assuming thermal equilibrium.

The ICM signatures provide additional sensitivity to the plasma composition through their amplitude and the frequencies at which they occur. For ion species with equal cyclotron frequencies the center frequencies of the ICM signatures coincide, and it is not possible to distinguish ions by the frequencies at which they create cyclotron features. However, the amplitudes and widths of the peaks are sensitive to the ion thermal velocity. In thermal equilibrium ions with different masses will therefore in addition to the different underlying widths of their contributions to $\left\langle\tilde{\mathbf{j}}^{(\mathrm{I} 0)} \tilde{\mathbf{j}}^{(\mathrm{I} 0)}\right\rangle-$ create ICM signatures with different amplitudes. When the ions have different cyclotron frequencies the ICM signatures increase the sensitivity to plasma composition even more. The ions then create ICM signatures in $\left\langle\tilde{\mathbf{j}}^{(\mathrm{I} 0)} \tilde{\mathbf{j}}^{(\mathrm{I} 0)}\right\rangle$ at their individual cyclotron harmonics, and the relative height of these features provides information on the relative densities. For example, in a plasma consisting of hydrogen and deuterium $\left(\omega_{\mathrm{cD}}=\omega_{\mathrm{cH}} / 2\right)$ there will be two sets of ICM signatures: one set with contributions from both hydrogen and deuterium at the frequencies where their harmonics coincide and one set resulting only from deuterium contributions. Thus, in figure 4a, ICM signatures resulting from deuterium alone can be seen between the features resulting from both hydrogen and deuterium - but only for low hydrogen ion density. At higher hydrogen densities $\left\langle\tilde{\mathbf{j}}^{(\mathrm{I} 0)} \tilde{\mathbf{j}}^{(\mathrm{I} 0)}\right\rangle$ is dominated by the hydrogen contribution, and this decreases the sensitivity to plasma composition.

Thus, figure 4 also shows an example of how ions which create strong currents and strong cyclotron features will tend to dominate the total $\left\langle\tilde{\mathbf{j}}^{(\mathrm{I} 0)} \tilde{\mathbf{j}}^{(\mathrm{I} 0)}\right\rangle$ even at relatively low densities. This affects the sensitivity to plasma composition: ions which create strong currents and cyclotron features can be detected even at low concentrations, but 
conversely they may also dominate the spectrum at high concentrations and render it less sensitive to the plasma composition. Figure $4 \mathrm{~b}$ shows contributions to $\left\langle\tilde{j}_{x}^{(\mathrm{IO})} \tilde{j}_{x}^{(\mathrm{IO})}\right\rangle$ from hydrogen, deuterium, tritium and helium. Hydrogen contributions are larger and have stronger cyclotron features than deuterium contributions which are in turn larger and have stronger cyclotron features than tritium contributions. However, the three hydrogen isotopes all give smaller contributions and weaker cyclotron features than helium. We can understand these properties from the explicit expressions for $\left\langle\tilde{\mathbf{j}}^{(\mathrm{i} 0)} \tilde{\mathbf{j}}^{(\mathrm{i} 0)}\right\rangle$, and since $\left\langle\tilde{j}_{x}^{(\mathrm{i} 0)} \tilde{j}_{x}^{(\mathrm{i} 0)}\right\rangle$ has the simplest analytic expression we shall consider this element in some detail. Carrying out the momentum integral in equation (11) and inserting in equation (10) we find

$$
\begin{aligned}
\left\langle\tilde{j}_{x}^{(\mathrm{i} 0)} \tilde{j}_{x}^{(\mathrm{i} 0)}\right\rangle(\mathbf{k}, \omega)= & \frac{2 \sqrt{\pi} q_{\mathrm{i}}^{2} n^{(\mathrm{i} 0)}}{v_{\mathrm{ti}}\left|k_{\|}\right|}\left(\frac{\omega_{\mathrm{ci}}}{k_{\perp}}\right)^{2} \\
& \times \sum_{l=-\infty}^{\infty} l^{2} \exp \left(-\zeta_{l}^{2}-k_{\perp}^{2} \rho_{\mathrm{i}}^{2}\right) I_{l}\left(k_{\perp}^{2} \rho_{\mathrm{i}}^{2}\right)
\end{aligned}
$$

For the parameter ranges considered here $k_{\perp}^{2} \rho_{\mathrm{i}}^{2}>20$ in the ion terms. In this case we can, to good accuracy, approximate the modified Bessel functions by $I_{\nu}(x) \rightarrow$ $\left.e^{x} / \sqrt{(} 2 \pi x\right), x \gg 1, \nu$ for the lower values of $l$. At low frequency shifts the higher harmonics contribute very little since the modified Bessel functions decay rapidly with increasing $l$. For low frequency shifts and using $\rho_{\mathrm{i}}^{2}=v_{\mathrm{ti}}^{2} / 2 \omega_{\mathrm{ci}}^{2}$ we then get

$$
\begin{aligned}
\left\langle\tilde{j}_{x}^{(\mathrm{i} 0)} \tilde{j}_{x}^{(\mathrm{i} 0)}\right\rangle(\mathbf{k}, \omega) & \cong \frac{2 q_{\mathrm{i}}^{2} n^{(\mathrm{i} 0)}}{v_{\mathrm{ti}}^{2}\left|k_{\|}\right|}\left(\frac{\omega_{\mathrm{ci}}}{\left|k_{\perp}\right|}\right)^{3} \times \sum_{l=-\infty}^{\infty} l^{2} \exp \left(-\zeta_{l}^{2}\right) \\
& =\frac{q_{\mathrm{i}}^{5}\left(B^{(0)}\right)^{3} n^{(\mathrm{i} 0)}}{m_{\mathrm{i}}^{2} T_{\mathrm{i}}\left|k_{\|}\right|\left|k_{\perp}\right|^{3}} \times \sum_{l=-\infty}^{\infty} l^{2} \exp \left(-\zeta_{l}^{2}\right)
\end{aligned}
$$

We can recognize some of the trends seen in figure 4 in the front factor of equation (15). Assuming thermal equilibrium, isotopes with larger mass will generally give lower contributions to $\left\langle\tilde{\mathbf{j}}^{(\mathrm{I})} \tilde{\mathbf{j}}^{(\mathrm{I} 0)}\right\rangle$ and ions with higher charge will give larger contributions. The precise relations depend on the ions involved. The front factor for deuterium will be four times smaller than that for hydrogen. On the other hand, the front factor for helium will be two times greater than that for hydrogen due to the greater charge.

In the limit $\phi \rightarrow 90^{\circ}$ the exponential function in the sum over $l$ will vary between unity at the cyclotron harmonics and zero everywhere else - as was discussed previously. The amplitude of the ICM signatures, which arise for $\phi \sim 90^{\circ}$, depends on how small the exponential function can be between the cyclotron harmonics (the maximum value, attained at the cyclotron harmonics, is always unity regardless of plasma parameters). The minima of $\left\langle\tilde{j}_{x}^{(\mathrm{i} 0)} \tilde{j}_{x}^{(\mathrm{i} 0)}\right\rangle$ are found at frequencies roughly halfway between cyclotron harmonics. At each of these minima the argument of the exponential function in the two leading terms in the sum over $l$ will be approximately

$$
\zeta_{l}^{2}=\frac{m_{\mathrm{i}}}{2 T_{\mathrm{i}}}\left(\frac{\omega_{\mathrm{ci}}}{2 k_{\|}}\right)^{2}=\frac{q_{\mathrm{i}}^{2} B^{2}}{8 m_{\mathrm{i}} T_{\mathrm{i}} k_{\|}^{2}} .
$$


The greater $\zeta_{l}^{2}$ is at the minima, the deeper the minima will be, and the greater the amplitude of the ICM signatures. So - as is also seen in figure 4 - assuming thermal equilibrium, isotopes with higher mass give cyclotron features with lower amplitudes. For constant charge to mass ratio the amplitude increases with increasing charge as is seen in figure 4 when comparing helium and deuterium.

\section{Signatures of ion Bernstein waves in the fluctuation operators}

The fluctuation operators quantify the plasmas dielectric response to current fluctuations driven by unscreened test particles moving along characteristics, $\tilde{\alpha}_{i}^{(\mathrm{a})}=$ $\hat{\mathbf{S}}_{i k}^{(\alpha \mathrm{a})} \tilde{j}_{k}^{(a 0)}$. To give an example, $\hat{\mathbf{S}}_{x z}^{(\mathrm{Ee})}$ gives the electric field fluctuation in the $x$-direction resulting from an electron current density fluctuation in the $z$-direction. The fluctuation operators are derived in [23] and are given by

$$
\begin{array}{ll}
\hat{\mathbf{S}}_{i k}^{(\mathrm{Ea})}=\frac{-i}{\omega \epsilon_{0}} \Lambda_{i k}^{-1}, & \hat{\mathbf{S}}_{i k}^{(\mathrm{Ba})}=\frac{-i k}{\omega^{2} \epsilon_{0}} \epsilon_{i j l} \hat{k}_{j} \Lambda_{l k}^{-1} \\
\hat{\mathbf{S}}_{i k}^{(\mathrm{ji})}=-\chi_{i j}^{(e)} \Lambda_{j k}^{-1}, & \hat{\mathbf{S}}_{i k}^{(\mathrm{je})}=\hat{\mathbf{S}}_{i k}^{(\mathrm{ji})}+\delta_{i k} \\
\hat{\mathbf{S}}_{k}^{(\mathrm{na})}=\frac{k_{i}}{\omega q_{e}} \hat{\mathbf{S}}_{i k}^{(\mathrm{ja})} &
\end{array}
$$

where

$$
\Lambda_{i j}=\epsilon_{i j}+N^{2}\left[\hat{k}_{i} \hat{k}_{j}-\delta_{i j}\right], \quad \epsilon_{i j}=\delta_{i j}+\sum_{a} \chi_{i j}^{(\mathrm{a})}
$$

is the plasma wave tensor and $\chi_{\mathrm{ij}}^{(\mathrm{a})}$ is the plasma susceptibility. The plasma susceptibility can show behaviors which are in some respects analogous to those studied for $\left\langle\tilde{\mathbf{j}}^{(\mathrm{i} 0)} \tilde{\mathbf{j}}^{(\mathrm{i} 0)}\right\rangle$ in section. 3. We will not give explicit expressions for every element in the plasma susceptibility, but to give an example it is instructive to examine the expression for $\chi_{\mathrm{xx}}^{(\mathrm{a})}$ which, using results from [26], can be written

$$
\begin{aligned}
\chi_{\mathrm{xx}}^{(\mathrm{a})}=\frac{2 \sqrt{\pi} q_{\mathrm{a}}^{2} n^{(\mathrm{a} 0)}}{k_{\perp}^{2} \rho_{\mathrm{i}}^{2} v_{\mathrm{ta}}\left|k_{\|}\right|} & \times\left[\sum_{l=-\infty}^{\infty} l^{2} D\left(\zeta_{l}\right) I_{l}\left(k_{\perp}^{2} \rho_{\mathrm{i}}^{2}\right) \exp \left(-k_{\perp}^{2} \rho_{\mathrm{i}}^{2}\right)\right. \\
& \left.+i \sqrt{\pi} \frac{k_{\|}}{\left|k_{\|}\right|} \sum_{l=-\infty}^{\infty} l^{2} \exp \left(-\zeta_{l}^{2}-k_{\perp}^{2} \rho_{\mathrm{i}}^{2}\right) I_{l}\left(k_{\perp}^{2} \rho_{\mathrm{i}}^{2}\right)\right]
\end{aligned}
$$

where $D(x)$ is the Dawson integral, which we note gives zero for $x=0$ and in the limits $x \rightarrow \pm \infty$. For $\phi \sim 90^{\circ}$ the sums appearing in equation (19) give the same type of oscillatory behavior near ion cyclotron harmonics as was found for $\left\langle\tilde{\mathbf{j}}^{(\mathrm{i} 0)} \tilde{\mathbf{j}}^{(\mathrm{i} 0)}\right\rangle$ in section. 3 - indeed, the sum in the imaginary part of $\chi_{\mathrm{xx}}^{(\mathrm{a})}$ is identical to the sum in equation (14) for $\left\langle\tilde{j}_{x}^{(\mathrm{a} 0)} \tilde{j}_{x}^{(\mathrm{a} 0)}\right\rangle$. However, the fluctuation operators depend on all elements of the plasma susceptibility through the inverse wave tensor. For $\phi \sim 90^{\circ}$ we may therefore expect the fluctuation operators to show an oscillatory behavior analogous, but not identical, to the behavior found for $\left\langle\tilde{\mathbf{j}}^{(\mathrm{i} 0)} \tilde{\mathbf{j}}^{(\mathrm{i} 0)}\right\rangle$. This behavior can be identified with the influence of weakly damped ion Bernstein waves on the plasma dielectric response to the unscreened current fluctuations, and its influence on the spectrum will be examined below. 


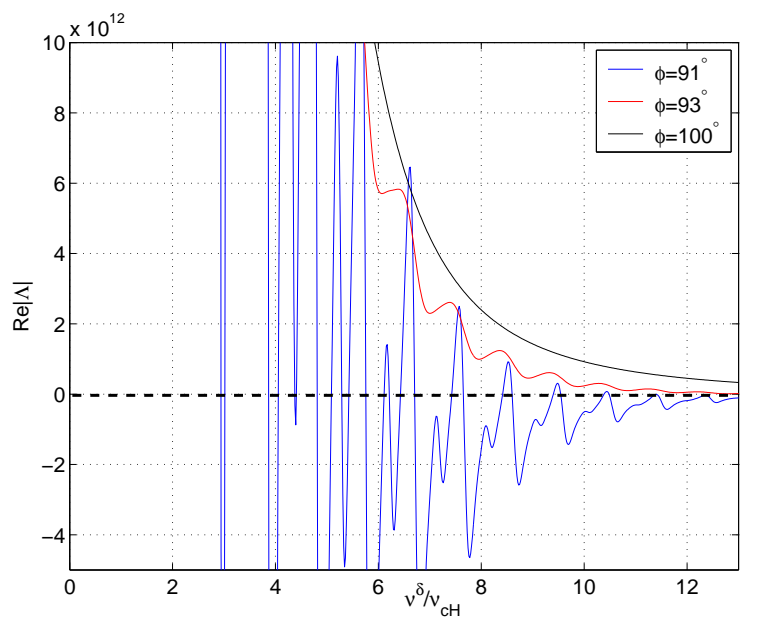

(a)

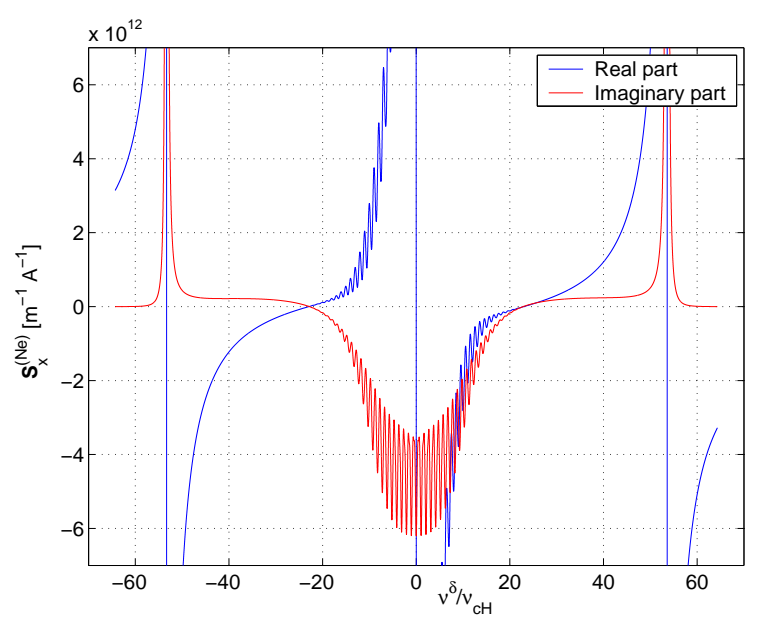

(b)

Figure 5. (a) $\operatorname{Re}(|\boldsymbol{\Lambda}|)$ in the standard plasma scenario, but for different values of $\phi$. For $\phi \sim 90^{\circ}$ weakly damped IBWs cause oscillations and eventually sign inversions in $\operatorname{Re}(|\boldsymbol{\Lambda}|)$ at frequencies $|\nu| / \nu_{\mathrm{cH}} \lesssim 20$. The fast magnetosonic wave causes a sign inversion at higher frequencies (not shown in the left graph, but the effects are seen on the right). (b) $\hat{\mathbf{S}}_{x}^{(\text {ne) }}$ for $\phi=93^{\circ}$ in the standard plasma scenario. The fast magnetosonic wave and IBWs cause enhanced dielectric response in their respective frequency ranges. The IBW signatures are poorly resolved on the frequency scale used here; they will be examined in greater detail below.

Plasma waves satisfy the dispersion relation $|\boldsymbol{\Lambda}|=0$ where $|\boldsymbol{\Lambda}|$ is the determinant of the wave tensor. At frequencies and wave vectors in the vicinity of such waves we expect the plasma dielectric response to unscreened thermal fluctuations to be strongly enhanced - with damped waves giving rise to weaker signatures covering broader frequency ranges than undamped waves. Each element in the fluctuation operators, equations (17), contains terms proportional to an element of the inverse wave tensor and thereby $\S$ to $|\boldsymbol{\Lambda}|^{-1}$ which will be nearly singular in the vicinity of a weakly damped wave. For scattering geometries with $\mathbf{k}^{\delta}$ close to or equal to the real part of the wave vector for a weakly damped wave, a peak will therefore occur in the spectrum near the wave frequency. This is illustrated in figure 5 which shows $\hat{\mathbf{S}}_{x}^{(\text {ne) }}$ and the real part of the determinant of the wave tensor for three different values of $\phi$ and with other parameters as in the plasma standard scenario.

For $\phi$ far from $90^{\circ}$, the real part of $|\boldsymbol{\Lambda}|$ is smooth and uniformly positive as a function of frequency. For $\phi$ approaching $90^{\circ}$ it starts to oscillate at low frequencies, and eventually changes sign (thus crossing zero) at frequencies close to the hydrogen cyclotron harmonics. For $\phi$ even closer to $90^{\circ}$ it crosses zero at the deuterium cyclotron harmonics as well. The imaginary part behaves in a qualitatively similar manner and is not shown - but we note that it does not cross zero at the same frequencies as the real part, which indicates that these are damped waves. For $\phi=90^{\circ}$ the real part of $|\boldsymbol{\Lambda}|$ has a singular behavior as it changes sign, and for high frequency shifts the sign inversions $\S$ From Cramer's rule for matrix inversion. 
take place very close to the cyclotron harmonics. Meanwhile the imaginary part goes to zero for $\phi=90^{\circ}$ and the waves are now undamped - except for collisional damping which is not included in the model used here. These waves are the pure ion Bernstein waves while the damped waves for $\phi \sim 90^{\circ}$ are neutralized ion Bernstein waves - neutralized because the electrons are able to stream along the magnetic field to neutralize the wave space charge. For fuel ion ratio diagnostics the neutralized ion Bernstein waves are the more relevant of the two, and they will be our main focus below. As expected we see in figure $5 \mathrm{~b}$ that the waves strongly enhance the fluctuation operator in the frequency range $|\nu| / \nu_{\mathrm{cH}} \lesssim 20$. Outside this range we note that the fast magnetosonic wave appears at frequencies around $|\nu| / \nu_{\mathrm{cH}} \simeq 53$ (for the standard scenario used here; the precise frequency depends on $\phi$ ). The wave tensor determinant, $\operatorname{Re}(|\boldsymbol{\Lambda}|)$, then changes sign again, and the wave causes a strong response in the fluctuation operators.

IBWs propagate at frequencies between harmonics of the cyclotron frequencies of each ion species in the plasma. Thus, in a pure hydrogen plasma the IBWs propagate between the hydrogen cyclotron harmonics. In a plasma with hydrogen and deuterium the dispersion relation changes to produce waves between each hydrogen harmonic and the neighboring deuterium harmonics. In plasmas with a larger number of ion species the picture becomes progressively more complicated, with each new species altering the dispersion relation for existing waves and giving rise to new waves if its cyclotron frequency does not coincide with those of the other ions. For the wave vectors considered here the frequencies will in practice be close to the ion cyclotron harmonics. Therefore each wave can, in a rough sense, be associated with a particular ion species, and the strength of its signature in the spectrum will increase with the density of that ion.

It is not possible to examine every element of the fluctuation operators in detail here. For simplicity we focus on the real part of $\hat{\mathbf{S}}_{x x}^{(\mathrm{Ea})}$, and figure 6 illustrates some key properties of its dependence on $R_{\mathrm{H}}=n_{\mathrm{H}} /\left(n_{\mathrm{H}}+n_{\mathrm{D}}\right)$. $\hat{\mathbf{S}}_{x x}^{(\mathrm{Ea})}$ was chosen only for ease in plotting as it is well behaved at $\nu^{\delta}=0$. The points discussed below apply to the fluctuation operators in general. Figure 6 a shows $\hat{\mathbf{S}}_{x x}^{(\mathrm{Ea})}$ for the standard plasma scenario and for plasmas dominated by respectively hydrogen and deuterium with the other parameters kept fixed. As expected the IBWs cause enhanced dielectric response at certain frequencies and at these frequencies peaks - or IBW signatures - appear in $\hat{\mathbf{S}}_{x x}^{(\mathrm{Ea})}$. Several properties of the IBW signatures are worth discussing. First, although the peaks appear at frequency intervals corresponding roughly to the ion cyclotron frequencies, they do not always appear at or even near the cyclotron harmonics. The IBW signatures originate from a dispersive wave, so in this respect their behavior can be very different from that of the ICM signatures which are always centered on the ion cyclotron harmonics. We also note in figure 6 that signatures of IBWs related to the presence of hydrogen tend to dominate. Clear signatures of IBWs related to deuterium are seen only in plasmas dominated by deuterium, and even then they are mainly seen near the lower cyclotron harmonics. This trend results from cyclotron damping of the IBWs. IBWs are affected by cyclotron damping when their frequencies fall in intervals roughly given by $\left|\omega / \omega_{\text {ci }}-n\right| \lesssim\left|k_{\|} \rho_{\mathrm{i}}\right|$ where $n$ is any positive integer. 


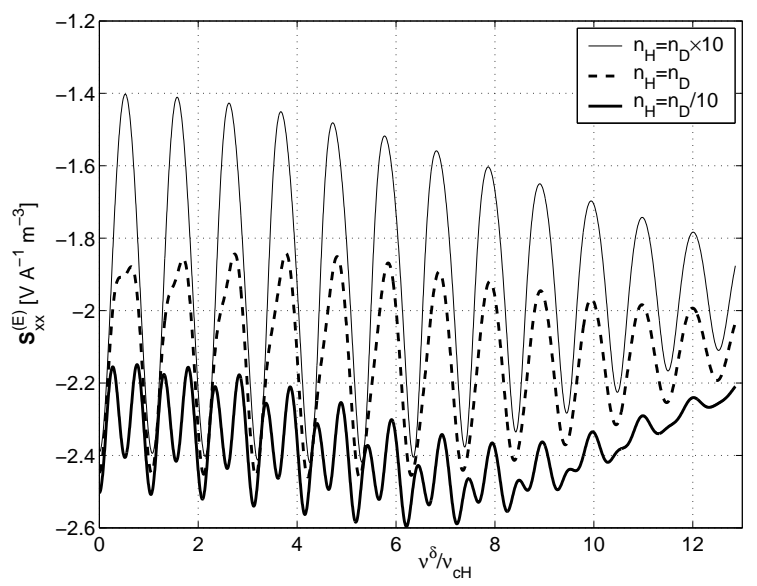

(a)

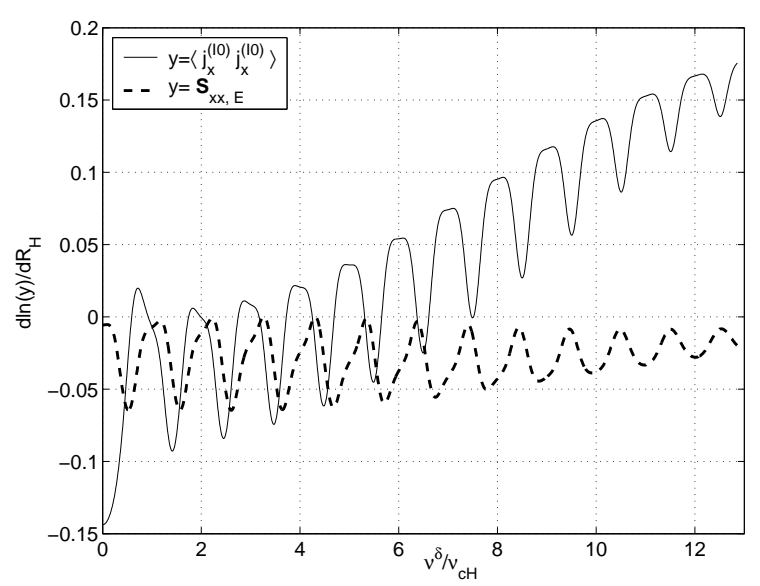

(b)

Figure 6. (a) The fluctuation operator $\hat{\mathbf{S}}_{x x}^{(\mathrm{E})}$ for the standard plasma scenario and for plasmas dominated by respectively hydrogen and deuterium with other parameters kept fixed. IBW signatures related to hydrogen dominate the operator except at low hydrogen density where signatures related to deuterium appear. (b) Derivatives of $\hat{\mathbf{S}}_{x x}^{(\mathrm{E})}$ and $\left\langle\tilde{j}_{x}^{(\mathrm{I} 0)} \tilde{j}_{x}^{(\mathrm{I} 0)}\right\rangle$ with respect to $R_{\mathrm{H}}$. Note that in the legend $\mathbf{S}_{\mathrm{xx}, \mathrm{E}}=\hat{\mathbf{S}}_{x x}^{(\mathrm{E})}$. To facilitate comparison of the two the figure shows the logarithmic derivative $\mathrm{d} \ln (y) / d R_{\mathrm{H}}=y^{-1} \mathrm{~d}(y) / d R_{\mathrm{H}}$. The very different functional dependence of the two quantities on $R_{\mathrm{H}}$ helps break degeneracies with other parameters.

For conditions relevant to the CTS measurements at TEXTOR we have $k_{\|} \rho_{\mathrm{H}} \sim 5 \cos \phi$. Cyclotron damping is therefore significant unless the resolved wave vector is nearly perpendicular to the magnetic field, and for practical purposes all the IBWs considered here are subject to some degree of cyclotron damping. Corresponding to the trend seen in figure 6, cyclotron damping will affect IBWs related to deuterium more strongly and over wider frequency ranges than it will affect IBWs related to hydrogen (assuming thermal equilibrium).

Although the wave damping decreases the strength of IBW signatures, we should note that it has certain useful features as well. Whereas the ICM signatures depend on ion charge and mass only in the combinations $T_{\mathrm{i}} / m_{\mathrm{i}}$ through the thermal velocity and $q_{\mathrm{i}} / m_{\mathrm{i}}$ through the cyclotron frequency, the cyclotron damping is fundamentally a finite Larmor radius effect which depends on the ratio $\sqrt{T_{\mathrm{i}} m_{\mathrm{i}}} / q_{\mathrm{i}}$. Thus, degeneracies in the functional dependence of the spectrum on the mass, charge and temperature of each ion can be resolved through the influence of wave damping.

The graph in figure $6 \mathrm{~b}$ shows the derivatives of $\left\langle\tilde{j}_{x}^{(\mathrm{I} 0)} \tilde{j}_{x}^{(\mathrm{I} 0)}\right\rangle$ and $\hat{\mathbf{S}}_{x x}^{(\mathrm{Ea})}$ with respect to $R_{\mathrm{H}}$. For easy comparison of these rather different quantities the figure shows the derivative normalized by the quantity itself, $\operatorname{dln}(y) / d R_{\mathrm{H}}=y^{-1} \mathrm{~d}(y) / d R_{\mathrm{H}}$. Here it is worth noting the signs, relative size and different shapes of the two derivatives.

In thermal equilibrium the velocity distribution for hydrogen is wider than that for deuterium. The derivative of any component in $\left\langle\tilde{\mathbf{j}}^{(\mathrm{I} 0)} \tilde{\mathbf{j}}^{(\mathrm{I} 0)}\right\rangle$ with respect to $R_{\mathrm{H}}$ will therefore be negative at low $\left|\nu^{\delta}\right|$ and positive at high $\left|\nu^{\delta}\right|$. Similarly, increases in the 
ion temperature have the general effect of increasing the average thermal velocity, so in the absence of ICM signatures the functional dependence of $\left\langle\tilde{\mathbf{j}}^{(\mathrm{I})} \tilde{\mathbf{j}}^{(\mathrm{I} 0)}\right\rangle$ on $T_{\mathrm{i}}$ and $R_{\mathrm{H}}$ can be very similar. However, all ICM signatures decrease in amplitude for increasing $T_{\mathrm{i}}$ (see the discussion of equation (16)) while some ICM signatures will increase and some will decrease in amplitude when $R_{\mathrm{H}}$ changes. This ability to break degeneracies in the functional dependence on different parameters significantly increases the diagnostic potential of spectra with ICM and IBW signatures.

In comparison with $\left\langle\tilde{\mathbf{j}}^{(\mathrm{I} 0)} \tilde{\mathbf{j}}^{(\mathrm{I} 0)}\right\rangle$ we see that the derivative of $\hat{\mathbf{S}}_{x x}^{(\mathrm{Ea})}$ stays negative at all frequencies. There are also differences with respect to the influence of ICM and IBW signatures on the two derivatives. Peaks in the derivatives do not occur at the same frequencies and they have different widths and amplitudes. The details are different for derivatives of other operators, but none of them closely reproduce derivatives of $\left\langle\tilde{\mathbf{j}}^{(\mathrm{I} 0)} \tilde{\mathbf{j}}^{(\mathrm{I} 0)}\right\rangle$. These differences in the functional dependencies on $R_{\mathrm{H}}$ further decrease the possibility that the functional dependence of the total spectrum on $R_{\mathrm{H}}$ will be degenerate with the dependence on other parameters. Therefore, while $\left\langle\tilde{\mathbf{j}}^{(\mathrm{I} 0)} \tilde{\mathbf{j}}^{(\mathrm{I} 0)}\right\rangle$ is in general more sensitive than the fluctuation operators to $R_{\mathrm{H}}$ and the amplitude of ICM signatures are in general greater than IBW signatures, it is not implied that $\left\langle\tilde{\mathbf{j}}^{(\mathrm{I} 0)} \tilde{\mathbf{j}}^{\mathrm{I} 0)}\right\rangle$ dominates the diagnostic potential. Indeed, we shall see below that for certain parameter ranges both effects are required to diagnose $R_{\mathrm{H}}$.

\section{Relative importance of ICM and IBW signatures for fuel ion ratio diagnostics}

Since both the fluctuation operators and the unscreened current correlation tensor display peaks for $\phi \sim 90^{\circ}$, it is relevant to consider the origin of peaks in the final spectrum - i.e., whether peaks in the spectrum are ICM or IBW signatures. However, with the exception of certain extreme cases it is not possible to give an unambiguous answer to this question, and in general the origin of the peaks will be mixed with contributions from both ICM and IBWs. Figure 7 seeks to illustrate this issue. Figure 7a shows the total scattering function and some of the more important elements in $\Sigma^{(\mathrm{e})}$ and $\Sigma^{(\mathrm{I})}$. The total scattering function is dominated by $\Sigma_{\mathrm{nn}}^{(\mathrm{I})}$, which describes scattering off density fluctuations. Figure $7 \mathrm{~b}$ compares $\Sigma_{\mathrm{nn}}^{(\mathrm{I})}$ to a few of the elements multiplied to calculate $\Sigma_{\text {nn }}^{(\mathrm{I})}$. Although the relative amplitudes of the ICM signatures in $\left\langle\tilde{\mathbf{j}}^{(\mathrm{I} 0)} \tilde{\mathbf{j}}^{\mathrm{I}(0)}\right\rangle$ are typically greater than the amplitudes of the IBW signatures in the fluctuation operators, it would be misleading to suggest that the ion cyclotron features dominate entirely. The combined peaks have a different shape and are often not centered on an ion cyclotron harmonic. Further, we should note from figure 7 a that $\Sigma^{(\mathrm{e})}$ contributes significantly to the total scattering function. Since $\left\langle\tilde{\mathbf{j}}^{(\mathrm{e} 0)} \tilde{\mathbf{j}}^{(\mathrm{e} 0)}\right\rangle$ does not contain ICM signatures, any peaks in $\Sigma^{(\mathrm{e})}$ result purely from IBW signatures in the fluctuation operators. For $\phi \sim 90^{\circ}$ the contribution of $\Sigma^{(\mathrm{e})}$ to the total scattering function is typically comparable to the ion terms, and it may even dominate for $T_{\mathrm{e}}>T_{\mathrm{i}}$. Therefore, even if the ICM signatures were the dominant cause of peaks in $\Sigma^{(\mathrm{I})}$, peaks in the total scattering function would 


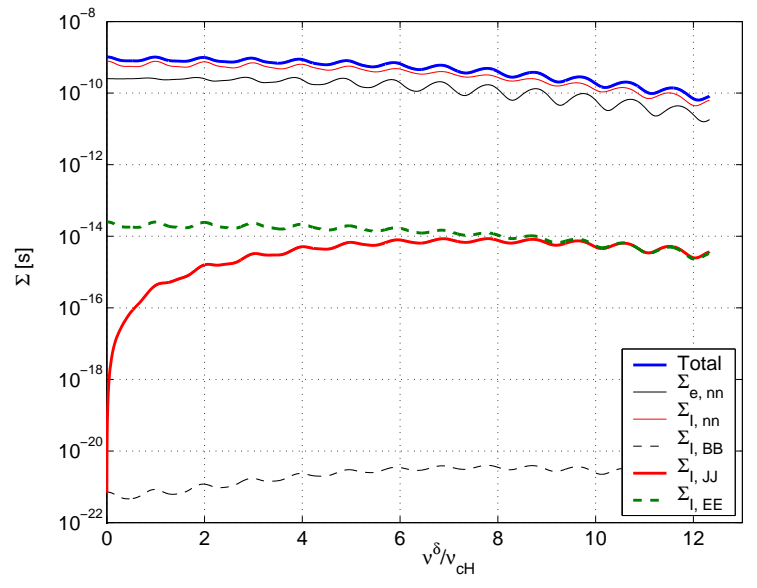

(a)

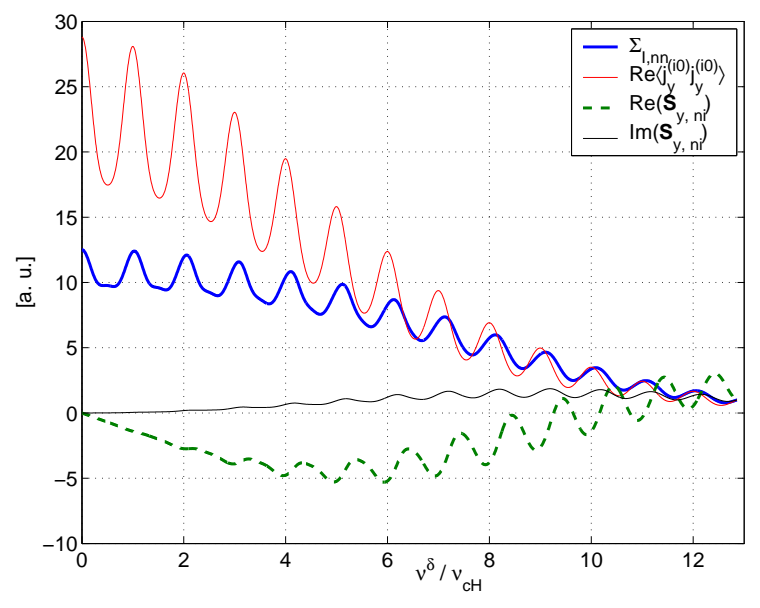

(b)

Figure 7. (a) Total scattering function for the standard scenario compared with $\Sigma_{\mathrm{nn}}^{(\mathrm{e})}$ and the diagonal elements of $\Sigma^{(\mathrm{I})}$. The scattering function is strongly dominated by the $\Sigma_{\mathrm{nn}}^{(\mathrm{I})}$ and $\Sigma_{\mathrm{nn}}^{(\mathrm{e})}$ elements. (b) The $\Sigma_{\mathrm{nn}}^{(\mathrm{I})}$ compared with $\left\langle\tilde{j}_{y}^{(\mathrm{I} 0)} \tilde{j}_{y}^{(\mathrm{I} 0)}\right\rangle$ and $\hat{\mathbf{S}}_{y}^{(\mathrm{ni})}-$ i.e., to some of the factors multiplied when calculating $\Sigma_{\mathrm{nn}}^{(\mathrm{I})}$. For comparison the quantities are scaled to fit on the same graph. Both the ICM and IBW signatures have a significant influence, and except in special cases it is in general not possible to say that peaks in the scattering function are dominated by a single effect. Note that in the legend $\mathbf{S}_{\mathrm{ij}, \alpha}=\hat{\mathbf{S}}_{i j}^{(\alpha)}$ and $\Sigma_{\mathrm{a}, \alpha \beta}=\Sigma_{\alpha \beta}^{(a)}$.

contain contributions from both effects.

To give an estimate of the relative importance of IBW and ICM signatures for fuel ion ratio measurements by CTS, we can perform a sensitivity analysis and thereby give theoretical estimates of the uncertainties of such measurements under different assumptions about ion magnetization. By calculating either $\left\langle\tilde{\mathbf{j}}^{(\mathrm{I} 0)} \tilde{\mathbf{j}}^{\mathrm{I} 0)}\right\rangle$ or the fluctuation operators with unmagnetized ions we find the theoretical uncertainty of the inferred values of $R_{\mathrm{H}}$ when the effects of the magnetic field are suppressed in either of the two terms. When the magnetic field is omitted in $\left\langle\tilde{\mathbf{j}}^{(\mathrm{IO})} \tilde{\mathbf{j}}^{(\mathrm{I} 0)}\right\rangle$ the spectrum will contain no ICM signatures. When ion magnetization is suppressed in the fluctuation operators the spectrum contains no signatures of IBWs (or other waves dependent on the magnetic field). By comparison of the resulting uncertainties we can then estimate the relative importance of each effect for the diagnostic potential.

We estimate the uncertainty of the inferred value of $R_{\mathrm{H}}$ within the framework of a Bayesian least squares method of inference [27] frequently used to interpret CTS measurements [7-12]. As we have seen above, the CTS spectrum depends in a non-trivial way - on a number of parameters besides the fuel ion ratio. The functional dependence on these parameters is taken into account in the analysis, and the Bayesian approach further allows prior knowledge from other diagnostics about all model parameters to be taken into account. The resulting posterior uncertainty on a given parameter (i.e., the state of knowledge after the measurement) therefore includes uncertainties in the prior information and further depends on the uncertainties in the 
CTS spectrum, and the Jacobian for the spectrum $[28,29]$. Within this framework and for given assumptions about the uncertainties in the prior information and the measured spectrum (i.e., the signal to noise ratio of the CTS receiver), the uncertainty of the inferred fuel ion ratio can therefore be estimated theoretically for a given set of plasma and system parameters.

Before discussing the results of these calculations we stress again that the outcome of the Bayesian analysis is an uncertainty which takes into account the assumed level of prior uncertainty for each parameter. Therefore the diagnostic potential of the measurement is expressed by the ratio between the prior and posterior uncertainties rather than by the posterior uncertainty alone. We shall assume a prior uncertainty (one standard deviation) of 0.5 for $R_{\mathrm{H}}$, and any posterior uncertainty below this value expresses a potential to increase knowledge about $R_{\mathrm{H}}$ through CTS measurements. We also stress that the uncertainties found with the approach taken here are basically an expression of the sensitivity of the spectrum to $R_{\mathrm{H}}$. They assume uncorrelated normally distributed noise levels in the data, and they assume that it is numerically possible to find the optimal fit to any measured spectrum (optimal in the least squares sense). Systematic errors resulting from inaccuracies in e.g. receiver calibration, background subtraction or the numerical optimization method are therefore not included. However, within these limits the analysis does provide some insight in the sensitivity of the spectrum to plasma composition, and here we use it to illustrate the effects of the different assumptions about ion magnetization.

Figure 8 shows results of such a sensitivity analysis for a scan of $\phi$ and $R_{\mathrm{H}}$. All calculations were done for the standard plasma parameters and the assumed prior uncertainties given in table A1 in Appendix A. The frequency resolution and bandwidth of the spectrum were taken to be those of the modified CTS receiver at TEXTOR. Similarly, the uncertainties in the measured spectra are assumed to be $2 \%$ of the signal strength as was found in initial experiments with the modified receiver and as is expected from theory [20].

When including effects of the magnetic field in all terms - i.e., with no simplifying assumptions - the posterior uncertainty is significantly reduced from the prior value when $\phi$ is close to $90^{\circ}$. As may be expected from the analysis in the preceding sections the uncertainty is lowest for low $R_{\mathrm{H}}$, but the ability to diagnose plasma compositions is by no means lost at high $R_{\mathrm{H}}$.

Comparing the result with unmagnetized $\left\langle\tilde{\mathbf{j}}^{(\mathrm{I} 0)} \tilde{\mathbf{j}}^{(\mathrm{I} 0)}\right\rangle$ to results with unmagnetized fluctuation operators, we generally find slightly greater uncertainties with unmagnetized $\left\langle\tilde{\mathbf{j}}^{(\mathrm{I} 0)} \tilde{\mathbf{j}}^{(\mathrm{I} 0)}\right\rangle$ (i.e., without ICM signatures). This shows that, at least in this special case, the ICM signatures are slightly more important for determination of $R_{\mathrm{H}}$ than the IBW signatures (and any other effect of the magnetic field in the fluctuation operators). However, to say that one of the two effects clearly dominates the diagnostic potential would be misleading. Especially at high $R_{\mathrm{H}}$ we see that in fact both effects are needed to diagnose plasma composition. This could indicate a degeneracy between the functional dependence of the spectrum on $R_{\mathrm{H}}$ and another parameter or set of parameters, which 


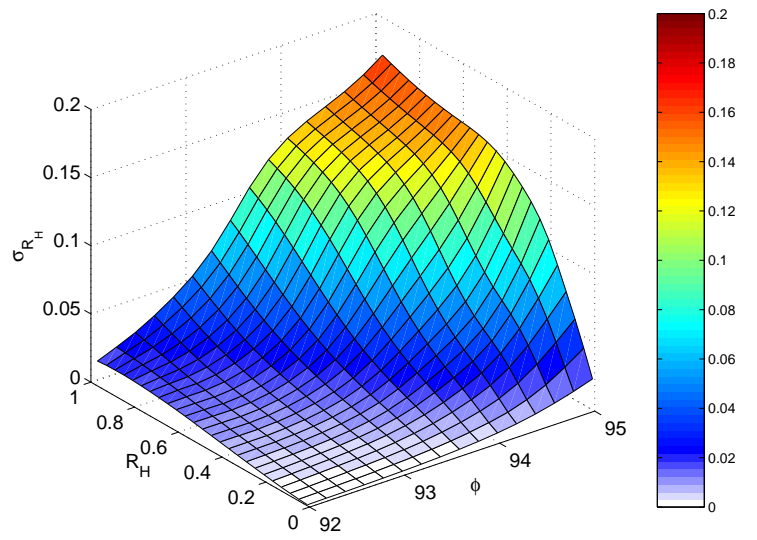

(a), $\sigma_{R_{\mathrm{H}}}$ including all effects of the magnetic field.

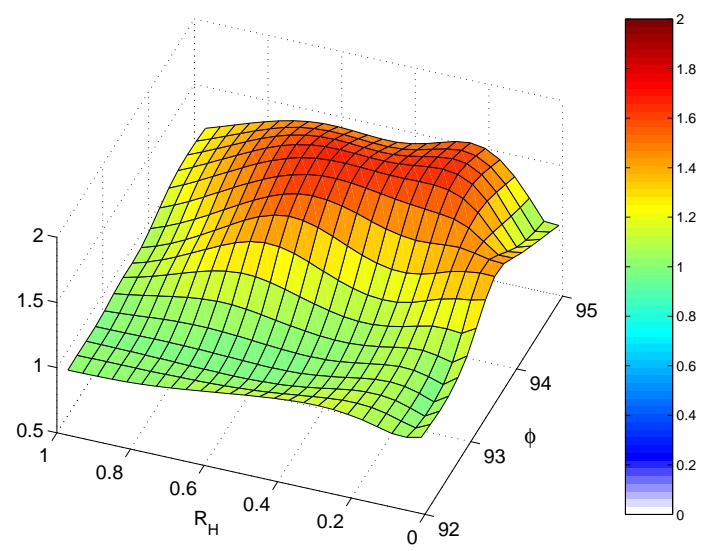

(d), ratio of uncertainties in the two approximations, $\sigma_{R_{\mathrm{H}}}^{(b)} / \sigma_{R_{\mathrm{H}}}^{(c)}$.

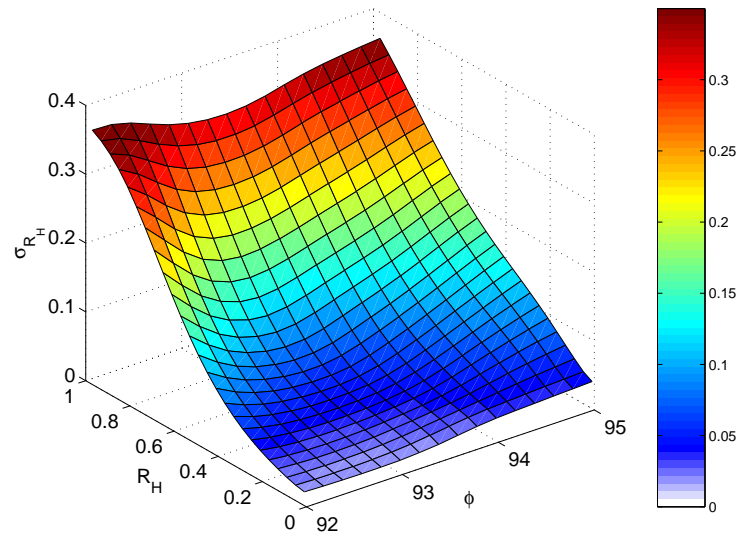

(b), $\sigma_{R_{\mathrm{H}}}^{(b)}$ calculated with unmagnetized $\left\langle\tilde{\mathbf{j}}^{(\mathrm{I} 0)} \tilde{\mathbf{j}}^{(\mathrm{I} 0)}\right\rangle$.

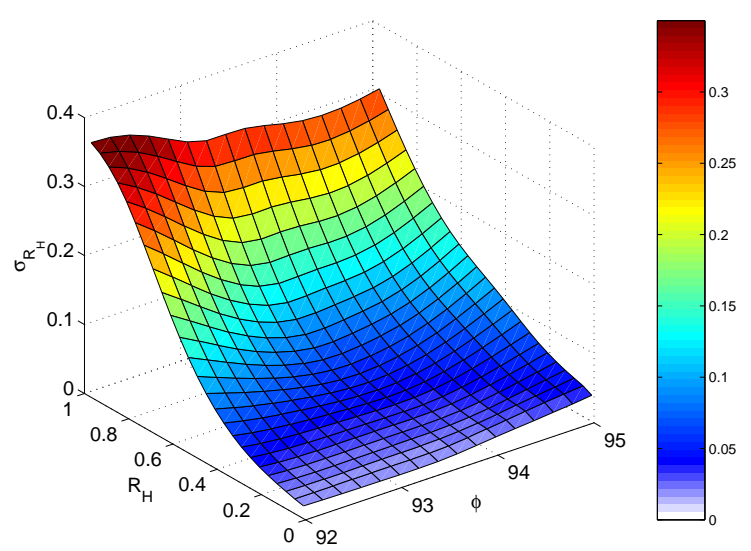

$(\mathrm{c}), \sigma_{R_{\mathrm{H}}}^{(c)}$ calculated with unmagnetized fluctuation operators.

Figure 8. Theoretically estimated uncertainties (one standard deviation) in $R_{\mathrm{H}}$ for scans of $\phi$ and $R_{\mathrm{H}}$ with different assumptions when calculating $\left\langle\tilde{\mathbf{j}}^{(\mathrm{i} 0)} \tilde{\mathbf{j}}^{(\mathrm{i} 0)}\right\rangle$ and the fluctuation operators. All parameters correspond to the standard plasma scenario with prior uncertainties as given in table A1. We caution that the uncertainties express the sensitivity of the spectrum to $R_{\mathrm{H}}$ and assume uncorrelated normally distributed prior uncertainties, and also that results would differ quantitatively for different plasma scenarios. Clockwise from the top left $(a)$, normal calculation including all effects. Top right $(b)$, results with unmagnetized ions when calculating $\left\langle\tilde{\mathbf{j}}^{\left({ }^{(10)}\right.} \tilde{\mathbf{j}}^{(\mathrm{I} 0)}\right\rangle$ - i.e., with IBW signatures, but no ICM signatures. Lower right $(c)$, results with unmagnetized ions when calculating the fluctuation operators - i.e., with no IBW signatures, but with ion cyclotron features. Lower left $(d)$, the ratio between the uncertainties with no cyclotron features and with no IBW signatures. The greatest uncertainties are found without ion cyclotron features, but in general both effects are seen to be important especially at high $R_{\mathrm{H}}$.

is resolved when both effects are included. 


\section{Conclusion}

We have presented a detailed study of the effects of ion cyclotron motion and ion Bernstein waves on the CTS spectrum with emphasis on the relevance of these effects for fuel ion ratio diagnostics. We have found that both effects appear when the underlying cyclotron structure of the CTS spectrum becomes noticeable, which happens for scattering geometries with resolved fluctuation wave vectors near perpendicular to the magnetic field, $\phi=\angle\left(\mathbf{B}^{(0)}, \mathbf{k}^{\delta}\right) \simeq 90^{\circ}$. For scattering with $\mathbf{k}^{\delta}$ at an oblique angle to the magnetic field, contributions to the spectrum from individual harmonics of the ion motion overlap and are smeared out in the total spectrum, but when the cyclotron motion is seen near right angles to the magnetic field, each harmonic contributes to the spectrum only in a narrow frequency range. The unscreened current correlation tensor, $\left\langle\tilde{\mathbf{j}}^{(\mathrm{I} 0)} \tilde{\mathbf{j}}^{(\mathrm{I} 0)}\right\rangle$, then displays ICM signatures while IBWs enter the spectrum through the fluctuation operators which, in the dressed particle approach to fluctuation theory, quantify the plasma dielectric response to the unscreened current fluctuations. Both the ICM and IBW signatures are sensitive to plasma composition as well as a range of other plasma parameters - perhaps most notably the ion temperature and impurity concentration which for simplicity have not been discussed in detail. While we have emphasized the diagnostic potential of the signatures for fuel ion ratio measurements, this emphasis should therefore not be taken to imply that this is the limit of their usefulness.

The shapes of contributions to $\left\langle\tilde{\mathbf{j}}^{(\mathrm{I} 0)} \tilde{\mathbf{j}}^{(\mathrm{I} 0)}\right\rangle$ from individual ions are not directly sensitive to the plasma composition, but the contribution from each ion is linearly proportional to the ion density, so the total $\left\langle\tilde{\mathbf{j}}^{(\mathrm{I} 0)} \tilde{\mathbf{j}}^{(\mathrm{I} 0)}\right\rangle$ is - under most conditions sensitive to the plasma composition. The ICM signatures in $\left\langle\tilde{\mathbf{j}}^{\mathrm{I}(0)} \tilde{\mathbf{j}}^{(\mathrm{I} 0)}\right\rangle$ increase the sensitivity to plasma composition, and they are especially useful in thermal equilibrium and for ions with different cyclotron frequencies. Some ions (generally those with low mass and high charge) give very strong contributions to $\left\langle\tilde{\mathbf{j}}^{(\mathrm{I} 0)} \tilde{\mathbf{j}}^{(\mathrm{I} 0)}\right\rangle$ and may therefore be detectable in small concentrations. Conversely, these ions may dominate entirely in high concentrations and render the spectrum less sensitive to plasma composition. The amplitude and width of cyclotron features vary with species, and generally ions with high charge and low mass give stronger cyclotron features.

Unlike the ICM signatures in $\left\langle\tilde{\mathbf{j}}^{(\mathrm{I} 0)} \tilde{\mathbf{j}}^{(\mathrm{I} 0)}\right\rangle$ the IBW signatures in the fluctuation operators reflect the influence of a dispersive wave. Although each wave and the strength of its signatures can roughly be associated with the presence of a particular ion species, their dispersion relation and the properties of their signatures depend on the total plasma state rather than that of a single ion population. Scattering from plasma fluctuations driven by the thermal motion of one particle species will therefore be affected by and carry information about all the other particle species in the plasma through the fluctuation operators. Perhaps the clearest example of this effect is that the electron contribution to the scattering function, $\Sigma^{(e)}$, displays IBW signatures and carries information about the relative ion densities. It is also noteworthy that, through 
the effects of wave damping, the IBW signatures introduce a functional dependence on the ion Larmor radius into the ion cyclotron structure. Since the ICM signatures are sensitive only to the thermal velocity and cyclotron frequency of the ions, the additional dependence on the ion Larmor radius can have the important effect of breaking degeneracies in the spectral variation with ion mass, charge and temperature.

Except for special cases, it does not appear possible to clearly separate the ICM and IBW signatures in the total spectrum. The terms producing the signatures are multiplied, so in general both effects will be present in the spectrum. An exception occurs when the electron temperature is much higher than the ion temperature. In this case scattering through $\Sigma^{(e)}$ dominates and since the electrons do not show ICM signatures the total spectrum will be dominated by IBW signatures.

We estimated the theoretical accuracy for measurements of $R_{\mathrm{H}}$ with the modified CTS receiver at TEXTOR through a sensitivity analysis within the framework of a Bayesian method of inference. The main purpose of this analysis was to illustrate the relative importance of ICM and IBW signatures for fuel ion ratio measurements, but we shall briefly comment the general results for the theoretical accuracy of $R_{\mathrm{H}}$. For the standard scenario used in this work, we find a theoretical accuracy of $\sigma_{\mathrm{H}}<0.05$ for all values of $R_{\mathrm{H}}$. In the preceding analysis we have found that IBW and ICM signatures related to hydrogen tend to dominate over those related to deuterium, and in the sensitivity analysis we find correspondingly that the highest accuracies may be expected for high deuterium density. However, the relative accuracy, $\sigma_{R_{\mathrm{H}}} / R_{\mathrm{H}}$ stays roughly constant as a function of $R_{\mathrm{H}}$. Results would differ quantitatively for other plasma scenarios, but these general trends would remain. Further, the conclusion that CTS measurements with ICM and IBW signatures may significantly increase knowledge about plasma composition has broad applicability beyond the plasma scenario assumed here. Initial studies [16] indicate that even in the presence of helium ash and other impurities, similar accuracies may be expected for ITER standard scenarios - which have very different plasma conditions from those assumed here, meaning that quantitative results should not be immediately carried over or extrapolated. A detailed study of the potential for fuel ion ratio diagnostics by CTS on ITER will be presented elsewhere.

By ignoring ion magnetization in the sensitivity analysis when calculating either $\left\langle\tilde{\mathbf{j}}^{(\mathrm{I} 0)} \tilde{\mathbf{j}}^{(\mathrm{I} 0)}\right\rangle$ or the fluctuation operators, the theoretical accuracy was calculated with either ICM or IBW signatures artificially suppressed. These approximations are not relevant for real data, but they allow an estimate of the relative importance of the two effects for fuel ion ratio measurements. The highest uncertainties were found without ICM signatures, but the difference between the two approximations was not significant. While ICM signatures are therefore slightly more important than IBW signatures for fuel ion ratio diagnostics it would be misleading to suggest that either of the two effects dominates the diagnostic potential. In fact, we also found that for high $R_{\mathrm{H}}$ the theoretical accuracy is greatly improved when both effects are included in the spectrum.

As final remark we note that the study presented here is relevant only to scattering 
from thermally excited plasma fluctuations with no external excitation mechanism. Signatures of externally driven ion Bernstein waves have been measured with far-infrared CTS in plasmas at Microtor [30,31], ACT-1 [3] and Alcator-C [32]. Cyclotron structure was also found in mm-wave CTS measurements of ion cyclotron resonance heated (ICRH) plasmas in the Tara Tandem Mirror axicell [33]. The structure was interpreted as IBW signatures, but from the analysis in this work we may expect that ICM signatures also contributed to the spectra - although we note that the effects of ICRH on the CTS spectrum remain unexplored in the analysis presented here. Measurements in a tokamak of thermally excited IBW and ICM signatures without external drive were demonstrated in recent results from TEXTOR [14]. The analysis presented here provides a framework for interpretation of such measurements, and the sensitivity analysis indicate that they have the potential to form the basis for a new fuel ion ratio diagnostic for fusion plasmas.

\section{Acknowledgments}

This work has been supported by the European Communities under the contract of Association between EURATOM and Risø DTU. It was carried out within the framework of the European Fusion Development Agreement under EFDA Contract WP08-09-DIA-01-05 (VI-1) Fuel ion ratio. The views and opinions expressed herein do not necessarily reflect those of the European Commission. 


\section{Appendix A. Parameter values used in numerical calculations}

Except where otherwise explicitly noted all numerical examples assume the parameters in table A1. These parameters are here referred to as the "standard scenario".

Table A1. Parameter values for the standard scenario used in numerical calculations. Prior uncertainties used in the sensitivity analysis in Section. $5, \sigma_{\text {prior }}$, are given in the last column. Prior uncertainties are not given for the ion densities, but instead for $R_{\mathrm{H}}$.

\begin{tabular}{llrll}
\hline Description & Symbol & Unit & Value & $\sigma_{\text {prior }}$ \\
\hline$\angle\left(\mathbf{k}^{\delta}, \mathbf{B}^{(0)}\right)$ & $\phi$ & Degrees & 93 & 3 \\
$\angle\left(\mathbf{k}^{\mathrm{i}}, \mathbf{k}^{\mathbf{s}}\right)$ & $\theta$ & Degrees & 159 & 3 \\
Frequency of incident radiation & $\nu^{\mathrm{i}}$ & $\mathrm{GHz}$ & 110 & \\
Mode of incident radiation & & & $\mathrm{O}$ & \\
Mode of scattered radiation & & & $\mathrm{O}$ & \\
Magnetic field strength & $B$ & $\mathrm{~T}$ & 2.6 & 0.1 \\
Electron density & $n_{\mathrm{e}}$ & $10^{19} \mathrm{~m}^{-3}$ & 2 & 0.3 \\
Hydrogen ion density & $n_{\mathrm{H}}$ & $10^{19} \mathrm{~m}^{-3}$ & 1 & \\
Deuterium ion density & $n_{\mathrm{D}}$ & $10^{19} \mathrm{~m}^{-3}$ & 1 & \\
Ion density ratio, $n_{\mathrm{H}} /\left(n_{\mathrm{H}}+n_{\mathrm{D}}\right)$ & $R_{\mathrm{H}}$ & & 0.5 & 0.5 \\
Electron temperature & $T_{\mathrm{e}}$ & $\mathrm{keV}$ & 1 & 0.4 \\
Ion temperature & $T_{\mathrm{i}}$ & $\mathrm{keV}$ & 1 & 0.4 \\
\hline
\end{tabular}

\section{References}

[1] A. J. H. Donné, A. E. Costley, R. Barnsley, H. Bindslev, R. Boivin, G. Conway, R. Fisher, R. Giannella, H. Hartfuss, M. G. Von Hellermann, E. Hodgson, L. C. Ingesson, K. Itami, D. Johnson, Y. Kawano, T. Kondoh, A. Krasilnikov, Y. Kusama, A. Litnovsky, P. Lotte, P. Nielsen, T. Nishitani, F. Orsitto, B. J. Peterson, G. Razdobarin, J. Sanchez, M. Sasao, T. Sugie, G. Vayakis, V. Voitsenya, K. Vukolov, C. Walker, K Young, and The ITPA Topical Group On Diagnostics. Chapter 7: Diagnostics. Nuclear Fusion, 47(6):S337-S384, June 2007.

[2] S. B. Korsholm, M. Stejner, S. Conroy, G. Ericsson, G. Gorini, M. Tardocchi, M. von Hellermann, R. J. E. Jaspers, O. Lischtschenko, E. Delabie, H. Bindslev, V. Furtula, F. Leipold, F. Meo, P. K. Michelsen, D. Moseev, S. K. Nielsen, and M. Salewski. Development of novel fuel ion ratio diagnostic techniques. Review of scientific instruments, 81(10):10D323, October 2010.

[3] G. Wurden, M. Ono, and K. Wong. Ion-temperature measurement via laser scattering on ion Bernstein waves. Physical Review A, 26(4):2297-2300, October 1982.

[4] H. Park, P. S. Lee, W. A. Peebles, and N. C. Luhmann. Tokamak ion temperature determination via cw far-infrared laser scattering. Review of Scientific Instruments, 56(5):922, 1985.

[5] R. Behn, D. Dicken, J. Hackmann, S. Salito, M. Siegrist, P. Krug, I. Kjelberg, B. Duval, B. Joye, and A. Pochelon. Ion temperature measurement of tokamak plasmas by collective Thomson scattering of D_\{2\}O laser radiation. Physical Review Letters, 62(24):2833-2836, June 1989.

[6] E. V. Suvorov, V. Erckmann, E. Holzhauer, W. Kasparek, Y. A. Dryagin, S. E. Fil'chenkov, A. A. Fraiman, T. Geist, M. Kick, L. M. Kukin, A. V. Kostrov, L. V. Lubyako, A. M. Shtanyuk, N. K. Skalyga, and O. B. Smolyakova. Ion temperature and beam-driven plasma waves from collective scattering of gyrotron radiation in W7-AS. Plasma Physics and Controlled Fusion, 37(11):1207-1213, 1995. 
[7] H. Bindslev, J. Hoekzema, J. Egedal, J. Fessey, T. Hughes, and J. Machuzak. Fast-Ion Velocity Distributions in JET Measured by Collective Thomson Scattering. Physical Review Letters, 83(16):3206-3209, October 1999.

[8] H. Bindslev, S. Nielsen, L. Porte, J. Hoekzema, S. Korsholm, F. Meo, P. Michelsen, S. Michelsen, J. Oosterbeek, E. Tsakadze, E. Westerhof, and P. Woskov. Fast-Ion Dynamics in the TEXTOR Tokamak Measured by Collective Thomson Scattering. Physical Review Letters, 97(20):205005, November 2006.

[9] H. Bindslev, S. K. Nielsen, L. Porte, J. A. Hoekzema, S. B. Korsholm, F. Meo, P. K. Michelsen, S. Michelsen, J. W. Oosterbeek, E. L. Tsakadze, E. Westerhof, and P. Woskov. Fast-ion dynamics in the TEXTOR tokamak measured by collective Thomson scattering. Plasma Physics and Controlled Fusion, 49(12B):B551-B562, December 2007.

[10] S. K. Nielsen, H. Bindslev, L. Porte, J. A. Hoekzema, S. B. Korsholm, F. Leipold, F. Meo, P. K. Michelsen, S. Michelsen, J. W. Oosterbeek, E. L. Tsakadze, G. Van Wassenhove, E. Westerhof, and P. Woskov. Temporal evolution of confined fast-ion velocity distributions measured by collective Thomson scattering in TEXTOR. Physical Review E, 77(1), January 2008.

[11] S. K. Nielsen, H. Bindslev, M. Salewski, A. Bürger, E. Delabie, V. Furtula, M. Kantor, S. B. Korsholm, F. Leipold, F. Meo, P. K. Michelsen, D. Moseev, J. W. Oosterbeek, M. Stejner, E. Westerhof, and P. Woskov. Fast-ion redistribution due to sawtooth crash in the TEXTOR tokamak measured by collective Thomson scattering. Plasma Physics and Controlled Fusion, 52(9):092001, September 2010.

[12] M. Salewski, F. Meo, M. Stejner, O. Asunta, H. Bindslev, V. Furtula, S.B. Korsholm, T. KurkiSuonio, F. Leipold, F. Leuterer, P.K. Michelsen, D. Moseev, S.K. Nielsen, J. Stober, G. Tardini, D. Wagner, and P. Woskov. Comparison of fast ion collective Thomson scattering measurements at ASDEX Upgrade with numerical simulations. Nuclear Fusion, 50(3):035012, March 2010.

[13] F. Meo, M. Stejner, M. Salewski, H. Bindslev, T. Eich, V. Furtula, S. B. Korsholm, F. Leuterer, F. Leipold, P. K. Michelsen, D. Moseev, S. K. Nielsen, B. Reiter, J. Stober, D. Wagner, P. Woskov, and The Asdex Upgrade Team. First results and analysis of collective Thomson scattering (CTS) fast ion distribution measurements on ASDEX Upgrade. Journal of Physics: Conference Series, 227:012010, May 2010.

[14] S.B. Korsholm, M. Stejner, H. Bindslev, V. Furtula, F. Leipold, F. Meo, P.K. Michelsen, D. Moseev, S.K. Nielsen, M. Salewski, M. de Baar, E. Delabie, M. Kantor, and A. Bürger. First measurements of intrinsic ion Bernstein waves in a tokamak by collective Thomson scattering. Accepted for publication in Physical Review Letters, 2011.

[15] R. K. Richards, D. P. Hutchinson, and C. H. Ma. Tritium to deuterium ratio measurement by collective Thomson scattering. Review of Scientific Instruments, 68(1):683, 1997.

[16] H. Bindslev, A. W. Larsen, F. Meo, P. Michelsen, S. Michelsen, A. H. Nielsen, S. Nimb, and E. Tsakadze. Final Report, EFDA Contract 04-1213 - deliverable 4.1-D3, ITER fast ion collective Thomson Scattering, 2005. Available at:

http://www.risoe.dk/

en/Research/sustainable_energy/Fusion_energy/projects/fusion_CTS/ITER.aspx

[17] F. Meo, H. Bindslev, S. B. Korsholm, E. L. Tsakadze, C. I. Walker, P. Woskov, and G. Vayakis. Design of the collective Thomson scattering diagnostic for International Thermonuclear Experimental Reactor at the $60 \mathrm{GHz}$ frequency range. Review of Scientific Instruments, 75(10):3585, 2004.

[18] M. Salewski, O. Asunta, L.-G. Eriksson, H. Bindslev, V. Hynönen, S. B. Korsholm, T. KurkiSuonio, F. Leipold, F. Meo, P. K. Michelsen, S. K. Nielsen, and J. Roenby. Comparison of collective Thomson scattering signals due to fast ions in ITER scenarios with fusion and auxiliary heating. Plasma Physics and Controlled Fusion, 51(3):035006, March 2009.

[19] M. Salewski, L.-G. Eriksson, H. Bindslev, S.B. Korsholm, F. Leipold, F. Meo, P.K. Michelsen, and S.K. Nielsen. Impact of ICRH on the measurement of fusion alphas by collective Thomson scattering in ITER. Nuclear Fusion, 49(2):025006, February 2009. 
[20] M. Stejner, S. K. Nielsen, S. B. Korsholm, M. Salewski, H. Bindslev, V. Furtula, F. Leipold, F. Meo, P. K. Michelsen, D. Moseev, A. Burger, M. Kantor, and M. de Baar. Collective Thomson scattering measurements with high frequency resolution at TEXTOR. Review of Scientific Instruments, 81(10):10D515, 2010.

[21] H. Bindslev. Dielectric effects on Thomson scattering in a relativistic magnetized plasma. Plasma Physics and Controlled Fusion, 33:1775, November 1991.

[22] H. Bindslev. Three-wave mixing and Thomson scattering in plasmas. Plasma Physics and Controlled Fusion, 35(11):1615-1640, 1993.

[23] H. Bindslev. A quantitative study of scattering from electromagnetic fluctuations in plasmas. $J$. Atmos. Terr. Phys., 58, 1996.

[24] N. C. Luhmann, H. Bindslev, H. Park, J. Sánchez, G. Taylor, and C. X. Yu. Chapter 3: Microwave Diagnostics. Fusion Science and Technology, 53:335, 2008.

[25] J. Sheffield. Plasma scattering of electromagnetic radiation. Academic Press, New York, 1975.

[26] D. G. Swanson. Plasma waves. Academic Press, Boston, MA (USA), 1989.

[27] H. Bindslev. Methods for optimizing and assessing diagnostic capability, demonstrated for collective Thomson scattering (invited). Review of Scientific Instruments, 70(1):1093, 1999.

[28] H. Bindslev. JET-R(97)13: Maximum likelihood estimators for inverse problems with nuisance parameters, 1997.

[29] M. Stejner, S. K. Nielsen, S. B. Korsholm, H. Bindslev, V. Furtula, F. Leipold, F. Meo, P. K. Michelsen, D. Moseev, and M. Salewski. Expected accuracy of fuel ion ratio measurements by collective Thomson scattering at TEXTOR. Submitted to EPS 2010, 2010.

[30] P. Lee, R. Taylor, W. Peebles, H. Park, C. Yu, Y. Xu, N. Luhmann, and S. Jin. Observation of Mode-Converted Ion Bernstein Waves in the Microtor Tokamak. Physical Review Letters, 49(3):205-208, July 1982.

[31] H. Park, N. Luhmann, W. Peebles, and R. Kirkwood. Observation of Second-Harmonic Ion Bernstein Waves Excited by Fast-Wave Mode Conversion in the Microtor Tokamak. Physical Review Letters, 52(18):1609-1612, April 1984.

[32] Y. Takase, J. Moody, C. Fiore, F. McDermott, M. Porkolab, and J. Squire. Study of directly launched ion Bernstein waves in a tokamak. Physical Review Letters, 59(11):1201-1204, September 1987.

[33] J. S. Machuzak, P. P. Woskov, R. C. Myer, W. J. Mulligan, D. R. Cohn, M. Gerver, S. N. Golovato, S. Horne, S. Kubota, R. S. Post, D. Rhee, J. Sullivan, and R. J. Temkin. Gyrotron collective Thomson scattering from plasma fluctuations in a Tara axicell. Review of Scientific Instruments, $59(8): 1562,1988$. 